\title{
Dynamics of Eddy-Driven Low-Frequency Dipole Modes. Part II: Free Mode Characteristics of NAO and Diagnostic Study
}

\author{
Dehai Luo and Tingting Gong \\ Physical Oceanography Laboratory, College of Physical and Environmental Oceanography, Ocean University of China, \\ Qingdao, China \\ ANTHONY R. LuPO \\ Department of Soil, Environmental and Atmospheric Sciences, University of Missouri-Columbia, Columbia, Missouri
}

(Manuscript received 8 December 2005, in final form 12 April 2006)

\begin{abstract}
Through calculating the scatter diagrams of the streamfunction $\left(\psi_{P}\right.$ or $\left.\psi_{T}\right)$ versus potential vorticity (PV) $\left(q_{P}\right.$ or $\left.q_{T}\right)$, where $\psi_{P}$ and $\psi_{T}$ are the planetary-scale streamfunction and total streamfunction, respectively, and using a weakly nonlinear NAO model proposed in Part I of this paper, it is suggested that negative- and positive-phase NAO events may approximately correspond to free modes even though driven by synopticscale eddies. In a planetary-scale field, the $q_{P}\left(\psi_{P}\right)$ scatter diagram of an NAO event exhibits a linear multivalued functional relationship in a narrow region for the negative phase, but exhibits a linear singlevalued functional relationship during the positive phase. It was also found that there is no steepening of the slope of the main straight line in the $q_{P}\left(\psi_{P}\right)$ scatter diagrams for two phases of the NAO event. Instead, the slope of the straight line in the scatterplots is time independent throughout the life cycle of the NAO event.

However, when synoptic-scale eddies are included in the streamfunction field, the $q_{T}\left(\psi_{T}\right)$ scatter diagram of the negative-phase NAO event shows a trend toward steepening during the intensification phase, and this tendency reverses during the decay phase. During the positive NAO phase the slope of the $q_{t}\left(\psi_{T}\right)$ scatter diagram shoals during the intensification phase and then steepens during the decay phase. Thus, it appears that the steepening and shoaling of the scatter diagrams of the streamfunction versus PV for the negativeand positive-phase NAO events are attributed to the effect of synoptic-scale eddies that force NAO events to form. Diagnostic studies using both composite and unfiltered fields of observed NAO events are presented to confirm these conclusions.
\end{abstract}

\section{Introduction}

The North Atlantic Oscillation (NAO) is one of the most prominent and recurrent patterns of lowfrequency atmospheric variability in the North Hemisphere (NH). The NAO not only affects the surface temperature and the occurrence of storms and precipitation over the Europe, but also influences the global climate (Hurrell 1995a). Although the NAO is found to exhibit remarkable decadal variability (Hurrell 1995a; Häkkinen 2000), the inherent time scale of the NAO events is actually about two weeks and the synoptic-

Corresponding author address: Dr. Dehai Luo, College of Physical and Environmental Oceanography, Ocean University of China, Qingdao 266003, China.

E-mail: 1dh@ouc.edu.cn

DOI: $10.1175 / J A S 3820.1$

(C) 2007 American Meteorological Society scale eddies may be the dynamical driver of the NAO (Feldstein 2003). In a previous paper (Luo et al. 2007, hereafter LLW), a weakly nonlinear model is proposed to account for the interaction between preexisting planetary-scale and synoptic-scale waves leads to an NAO event, a zonally localized dipole mode (Hurrell 1995b; Vallis et al. 2004; Cash et al. 2005). Results from this theoretical model are found to bear a striking resemblance to observed NAO events for the negative and positive phases (Benedict et al. 2004). It is shown that the phase of the NAO event depends strongly upon whether the preexisting planetary waves and preexisting eddy forcing (PEF) from synoptic-scale waves have a matching spatial structure. During the life cycle of the NAO, the PEF is found to be able to play either a positive role in growing the NAO or a negative role that leads to NAO decay. Since there is broad and 
growing interest in the NAO, exploring the physical dynamics of eddy-driven NAO events is a research question of critical importance. In this paper, we attempt to confirm the viewpoint that eddy-driven NAO events with a period of about two weeks can correspond approximately to free modes.

In the weakly nonlinear model proposed by LLW, dipole solutions that describe the life cycles of NAO events for positive and negative phases could be found. The negative-phase NAO solution corresponds to the solution of dipole blocking events occurring in the Atlantic sector, but the positive-phase solution corresponds to an intensified jet anomaly, which is found in the Pacific (Dole and Gordon 1983). Haines et al. (1993) showed that the dipole blocking anomalies become strongly nonlinear at upper levels as the potential vorticity contours close off. In this case, the modon model may be more appropriate. The weakly nonlinear mechanism, however, may be more important for the intensified jet anomalies (positive-phase NAO events) because their potential vorticity contours do not become closed at any stage.

In LLW, a weakly nonlinear model was proposed as a unified model for observed NAO events for both positive and negative phases. A comparison between observed NAO and blocking events indicated that the negative-phase NAO event is actually identical to blocking events in the Atlantic sector because there is a large similarity between the negative-phase NAO and blocking events in many aspects (period, pattern, and driving mechanism) (Berggren et al. 1949; Benedict et al. 2004; LLW). Thus, it was concluded that the negative-phase of the NAO event $\left(\mathrm{NAO}^{-}\right)$is a free mode and could be described by the weakly nonlinear model in LLW in that the blocking events can exhibit local free-mode dynamics (Butchart et al. 1989; Ek and Swaters 1994). In a quasigeostrophic framework, a free mode can be characterized formally by the functional relation $q=q(\psi)$ (Read et al. 1986; Branstator and Opsteegh 1989; Butchart et al. 1989; Anderson 1992; Ek and Swaters 1994), where $\psi$ is the streamfunction, and $q$ is the potential vorticity (PV). For a free mode, the $q(\psi)$ scatter diagrams collapse onto a straight line. Notwithstanding the mature $\mathrm{NAO}^{-}$event can be regarded as a free mode as in previous studies, how the free mode dynamics of the $\mathrm{NAO}^{-}$event behaves depends upon the stage of its life cycle. Whether the positive phase of the NAO $\left(\mathrm{NAO}^{+}\right)$event represents a free mode at all is unclear in theoretical and observational aspects. Then, the aim of the present paper is to construct the scatter diagrams of the streamfunction versus PV from the theoretical model, observed NAO cases, and composite NAO fields in order to demonstrate that observed NAO events can approximately correspond to free modes.

This paper is organized as follows: In section 2, the scatter diagrams are calculated based upon the planetary-scale fields of $\mathrm{NAO}^{-}$and $\mathrm{NAO}^{+}$events shown in LLW. In addition, a planetary-scale NAO event resembling an omega-type block is presented as in Luo and Chen (2006). In section 3, the scatter diagrams of the total streamfunction against its corresponding PV are presented to theoretically verify that the steepening (shoaling) and shoaling (steepening) of the scatter diagrams during the life cycle of the $\mathrm{NAO}^{-}\left(\mathrm{NAO}^{+}\right)$ events are strongly associated with upstream synopticscale eddies. In section 4, we present a diagnostic study of the scatter diagrams of streamfunction versus PV using two composite fields of $\mathrm{NAO}^{-}$and $\mathrm{NAO}^{+}$events using the daily NAO index of Benedict et al. (2004). In section 5 , the scatter diagrams of the streamfunction versus PV for unfiltered fields of two NAO cases are computed. In section 6, a strongly nonlinear numerical experiment performed by Haines and Malanotte- Rizzoli (1991, hereafter HMR) is briefly described in order to demonstrate that the theoretical NAO model proposed in LLW can describe free mode characteristics of observed NAO events even though this theoretical model is weakly nonlinear. The conclusion and discussions are summarized in section 7 .

\section{Free mode dynamics of NAO events in a theoretical model}

In the diagnostic studies of split jet and intensified jet events, Butchart et al. (1989), HMR, and Haines et al. (1993) suggested that the free mode characteristics of persistent structures described by theories are, to a large extent, determined by the form of the potential function $\Lambda=d q / d \psi$. In this paper, diagnostic studies of the features of the scatter diagram $(\Lambda=d q / d \psi)$ with and without synoptic-scale eddies included will be performed in order to understand if eddy-driven NAO events for positive and negative phases are free modes. In addition, the $\mathrm{NAO}^{-}$and $\mathrm{NAO}^{+}$events obtained in LLW will be used to compute the scatter diagrams of $q$ versus $\psi$ so as to make a comparison with diagnostic studies from data.

\section{a. Scatter diagrams of negative-phase NAO events and free modes}

\section{1) Dipole-TyPe EVEnTS}

Figure 1 shows the planetary-scale fields of a $\mathrm{NAO}^{-}$ event driven by synoptic-scale eddies through the interaction with the preexisting planetary wave, as in LLW 
(day 0 )

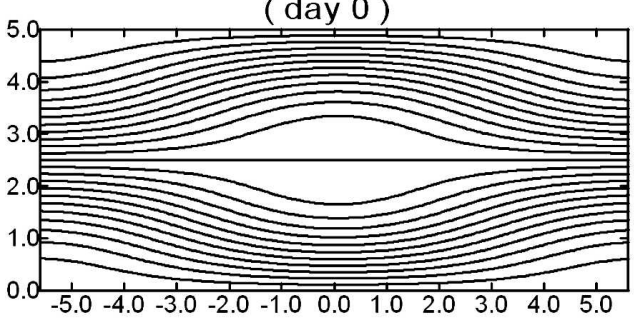

(day 6 )

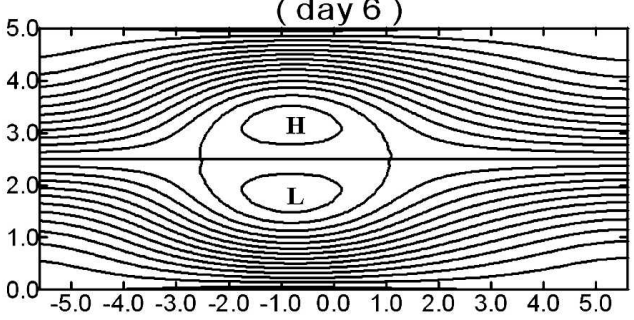

(day 12 )
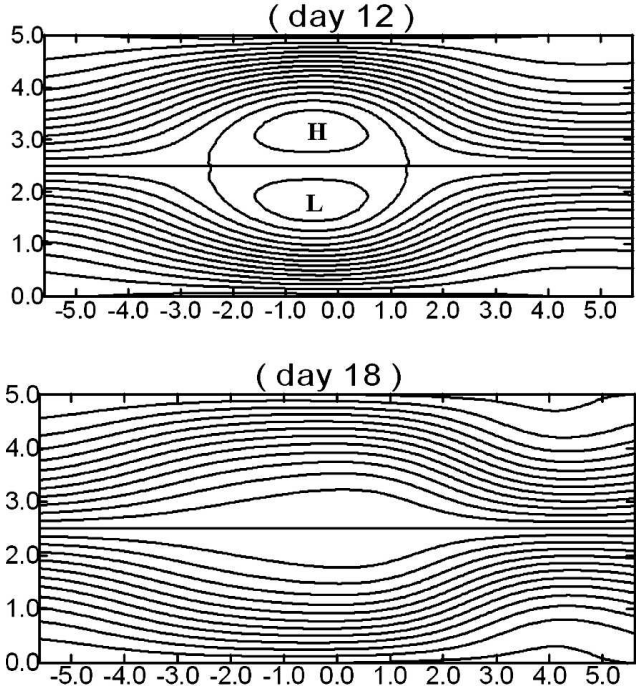

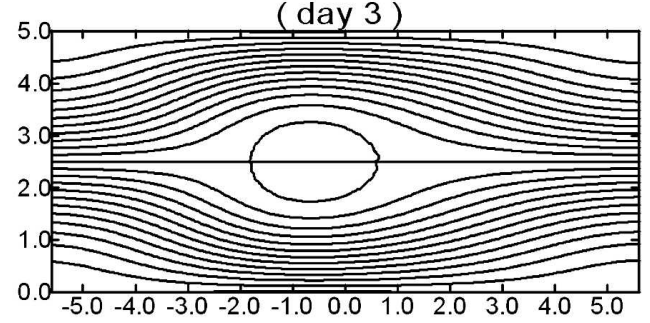

(day 9 )

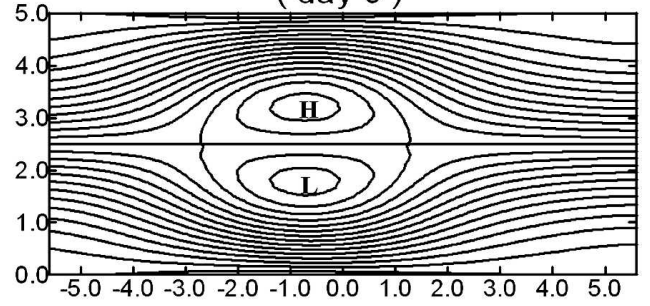

(day 15 )

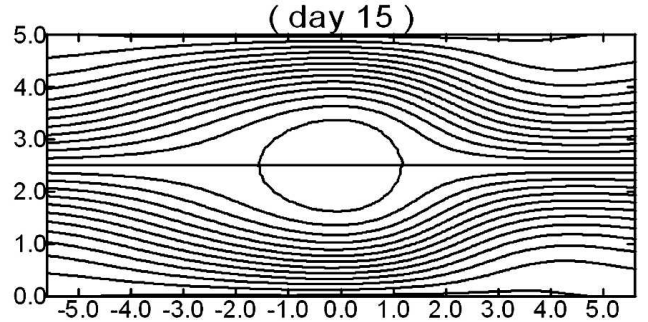

(day 21 )

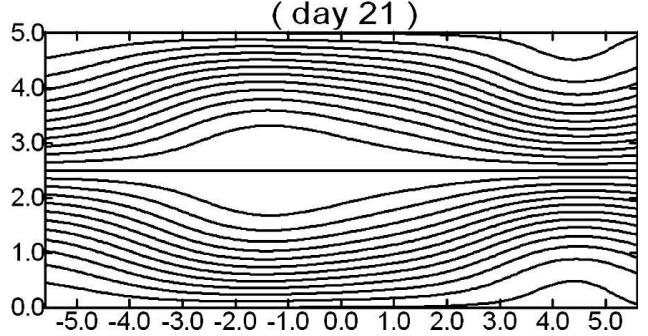

FIG. 1. Planetary-scale fields for the life cycle of a negative-phase NAO event, in which the contour interval (CI) is 0.3 (from LLW).

(their Fig. 3a). It was found that in the planetary-scale field, the life cycle of the $\mathrm{NAO}^{-}$event is similar to that of a dipole block, a high-over-low dipole height anomaly. In the total field, however, there appears to be several isolated cyclonic and anticyclonic vortices coexisting within the NAO region (LLW, their Fig. 3c). This implicates that negative-phase NAO events observed in the real atmosphere looks like a meandering blocking flow (Berggren et al. 1949; Benedict et al. 2004; LLW).

In Fig. 1, the negative-phase NAO anomaly at the mature stage (day 9) is strongly nonlinear because its potential vorticity contours close off completely in this stage (McWilliams 1980; HMR). In this case, the strongly nonlinear modon model of McWilliams (1980) may be more appropriate. Although the amplitude of the mature NAO anomaly is beyond the strict range of validity of the weakly nonlinear theory, a comparison with the scatter diagrams in a numerical experiment by HMR in a strongly nonlinear limit suggests that the weakly nonlinear model in LLW approximately represents strongly nonlinear coherent structures. An example of this type of behavior might be isolated split jet anomalies, even though the weakly nonlinear process may be dominant in the early and decay stages of coherent structures. Diagnostic studies are needed in order to determine whether the weakly nonlinear dynamics is relevant to negative- and positive-phase NAO anomalies. A useful method for testing this theory is to plot the scatter diagrams of the streamfunction versus PV from both data and the model, and then to perform a comparison. Before a diagnostic study is performed, 
the scatter diagrams of the streamfunction versus PV in the weakly nonlinear model proposed by LLW will be presented.

Figure 2 shows the scatter diagrams of the planetaryscale streamfunction against its corresponding PV from $-2 \leq x \leq 2$ during the life cycle of the $\mathrm{NAO}^{-}$event shown in Fig. 1. It was found that for an $\mathrm{NAO}^{-}$event the scatter diagram is time dependent and reveals a multivalued functional relationship in a narrow region during the period from day 3 to 15 , although the main functional line on the plot exhibits a straight line. Thus, it is natural to conclude that the $\mathrm{NAO}^{-}$events are a free mode because the multivalued functional relationship of the scatter diagram is a manifestation of strongly nonlinear isolated structures. This behavior has been demonstrated by many investigators (McWilliams 1980; Butchart et al. 1989). Of course, $\mathrm{NAO}^{-}$events can also be approximated as a free mode in that the deviation from the straight line of $q_{P}=q_{P}\left(\psi_{P}\right)$ is rather small.

To see the dependence of the multivalued functional lines of $q_{P}$ versus $\psi_{P}$ on the initial value (intensity and structure) of the $\mathrm{NAO}^{-}$event, we can consider $B(x, 0)=$ $B_{0} \cos (\varepsilon x)$ and $B(x, 0)=B_{0} \operatorname{sech}\left(\sqrt{\delta /(2 \lambda)} B_{0} x\right)$ having $B_{0}=0.35$ and $\varepsilon=0.24$ as two initial values for the theoretical NAO event in LLW to examine this problem. Figure 3 shows the scatterplots of $q_{P}$ versus $\psi_{P}$ at day 9 for the two initial values. It is found that the different choice of the initial value of the negativephase NAO does not affect the multivalued functional relationship in the scatter diagrams of $q_{P}$ versus $\psi_{P}$ during its mature stage. In other words, such a characteristic of the scatter diagrams may be the essence of the $\mathrm{NAO}^{-}$event, indicating that our weakly nonlinear model can, to a certain extent, describe the strongly nonlinear local free-mode characteristics of $\mathrm{NAO}^{-}$ events even if excited by synoptic-scale eddies (Butchart et al. 1989; HMR).

\section{2) OMEGA-TYPE EVENTS}

In the real atmosphere, an $\mathrm{NAO}^{-}$event in a planetary-scale field does not exactly exhibit the high-overlow (dipole) structure, as shown in Fig. 1. It sometimes possesses an Omega-type blocking structure in a composite field (Benedict et al. 2004, their Fig. 5). Figure 4 shows the planetary-scale field of a negative-phase NAO event driven by both synoptic-scale eddies and large-scale land-sea contrast (LSC) topography for the initial value of $B(x, 0)=B_{0} \cos (\varepsilon x)$ for $B_{0}=0.3, \varepsilon=$ 0.24 , and $h_{0}=0.5$ (a topographic parameter) in the weakly nonlinear blocking model proposed in Luo and Chen (2006), but with other parameters the same as in Fig. 1.

It is interesting to note that in the presence of LSC topography the negative-phase NAO event driven by synoptic-scale eddies has an Omega type blocking structure in a planetary-scale field. This shows that the LSC topography may play a certain role in the formation of realistic $\mathrm{NAO}^{-}$patterns. Of course, the strength and detailed shape of the $\mathrm{NAO}^{-}$event in a planetaryscale field is found to depend upon the initial conditions, the setting of the background westerly wind, and other factors, as pointed out in LLW (not shown). As we will find in the next section, the composite field of observed $\mathrm{NAO}^{-}$events indeed exhibits an Omega type blocking structure. Thus, it is possible to speculate that Fig. 4 looks more similar to the structure of composite $\mathrm{NAO}^{-}$events.

To examine if the scatter diagram of planetary-scale streamfunction against its corresponding PV depends upon the shape of observed $\mathrm{NAO}^{-}$events, the $q_{P}\left(\psi_{P}\right)$ scatter diagrams at different stages of an $\mathrm{NAO}^{-}$event shown in Fig. 4 are also calculated and shown in Fig. 5.

It was found in Fig. 5 that in the presence of LSC topography there is still a multivalued functional relationship of the $q_{P}\left(\psi_{P}\right)$ scatter diagram in a narrow region for an Omega type NAO structure, which is particularly noticeable at the mature stage, but two straight lines in this scatterplot are not more evident than in the dipole NAO event shown in Fig. 2.

\section{b. Scatter diagrams of positive-phase NAO events and free modes}

Figure 6 shows the planetary-scale fields of an $\mathrm{NAO}^{+}$ event driven by synoptic- scale eddies, which is cited from LLW. Note that the $\mathrm{NAO}^{+}$event in a planetaryscale field is represented entirely by a low-over-high dipole anomaly (nonblocking). This is actually similar to a jet intensification anomaly over the North Pacific observed by Dole and Gordon (1983) and Dole (1986). HMR showed that the jet intensification anomaly is weakly nonlinear even in the high amplitude regime because the potential vorticity contours do not, in general, become closed at any stage. It was found numerically that the scatter diagram of $q_{P}$ versus $\psi_{P}$ for the intensified jet anomaly at the mature stage exhibits a linear single-valued functional relationship. Here we will further calculate the scatter diagrams of $q_{P}$ versus $\psi_{P}$ during the life cycle of an $\mathrm{NAO}^{+}$event from both theoretical and observational point of view in order to ascertain whether the weakly nonlinear model proposed in LLW is able to describe the free mode characteristics of $\mathrm{NAO}^{+}$events.

The scatter diagrams of $q_{P}$ versus $\psi_{P}$ in the region of $-2 \leq x \leq 2$ during a life cycle of the $\mathrm{NAO}^{+}$event as described in Fig. 6 are shown in Fig. 7. It is found that the points on the scatter diagram almost congregate in 

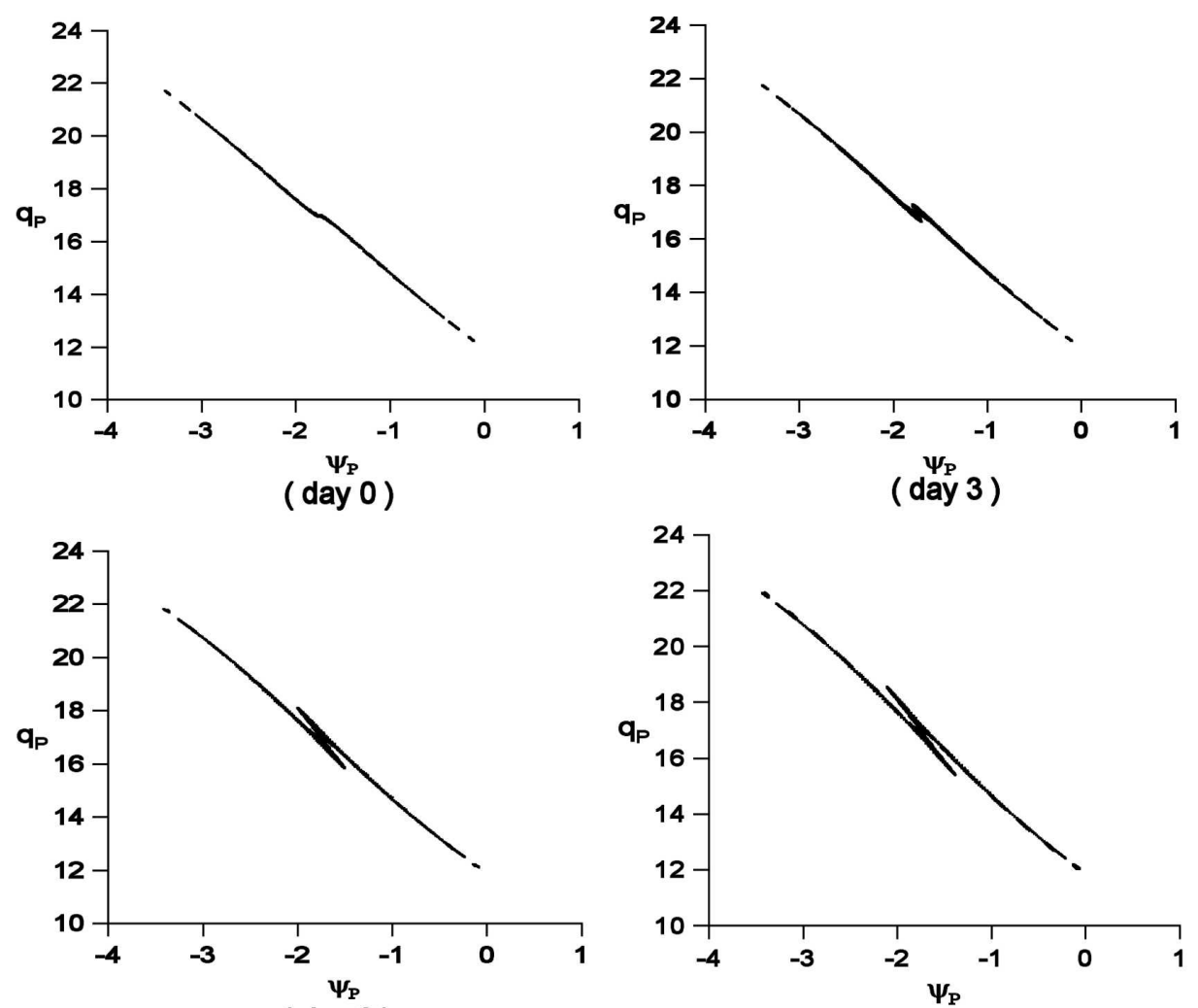

( day 6)

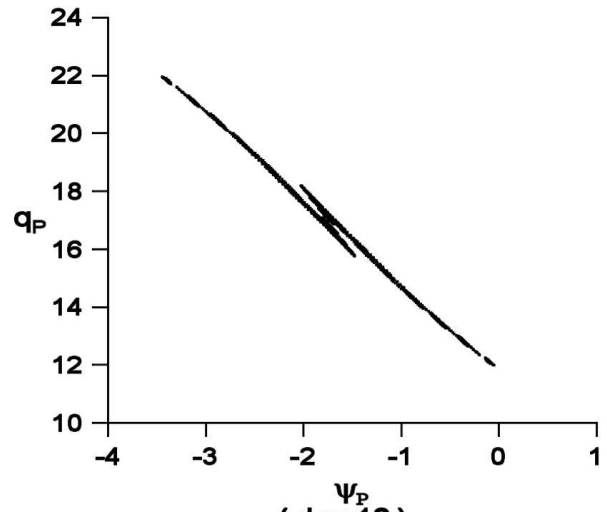

( day 9)

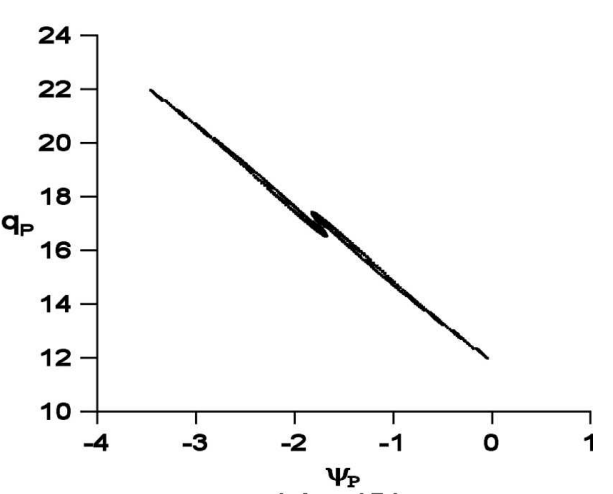

( day 12 )

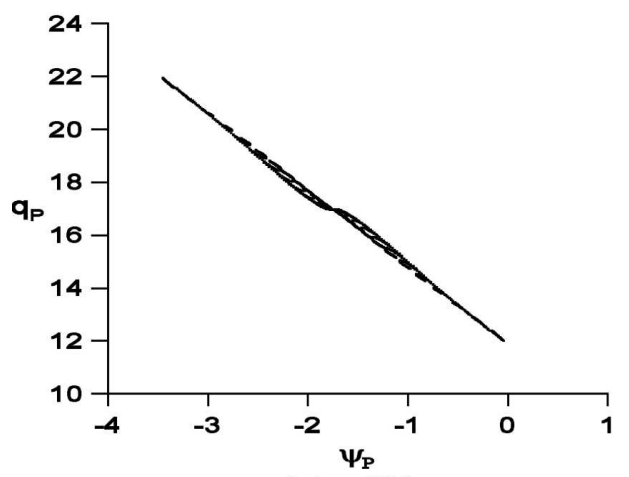

( day 18)

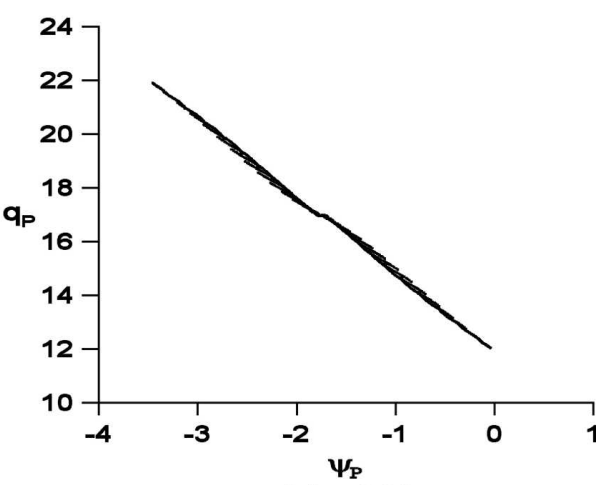

( day 21 )

FIG. 2. Scatter diagrams of the planetary-scale streamfunction $\left(\psi_{P}\right)$ vs its corresponding PV $\left(q_{P}\right)$ for the life cycle of the negative-phase event shown in Fig. 1. 
(a)

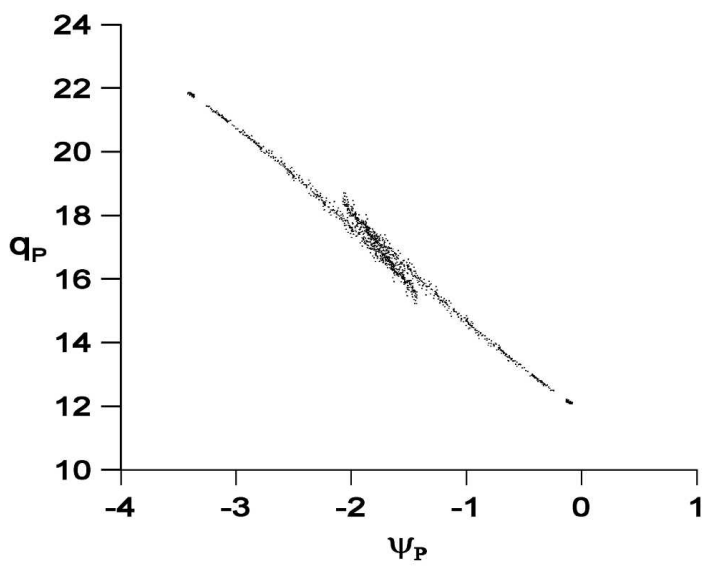

(b)

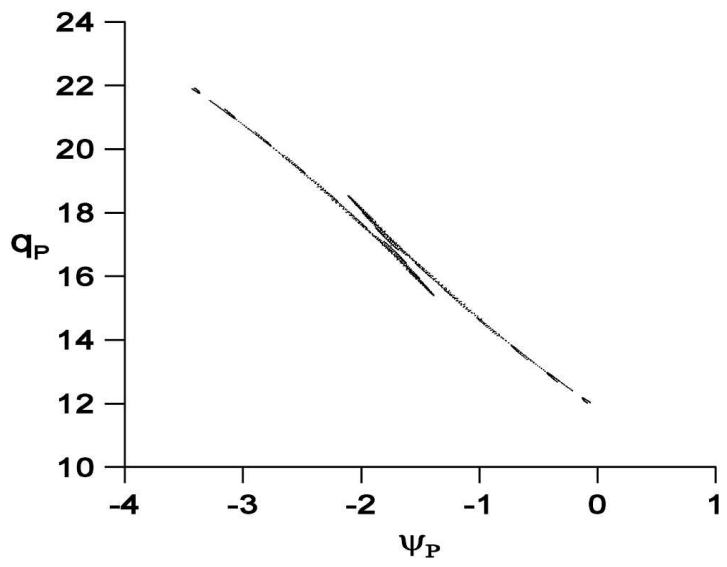

FIG. 3. Scatter diagrams of $\psi_{P}$ vs $q_{P}$ of a negative-phase NAO event at day 9 for two different initial values: (a) initial value $B(x$, $0)=B_{0} \cos (\varepsilon x)$ for $B_{0}=0.35$ and $\varepsilon=0.24$, and (b) initial value $B(x, 0)=B_{0} \operatorname{sech}\left(\sqrt{\delta /(2 \lambda)} B_{0} x\right)$ for $B_{0}=0.35$.

a straight line. The main straight line in this scatterplot is found to be time-independent, but the detail of the scatterplots is time dependent. For example, at the mature stage the slope $d q_{P} / d \psi_{P}$ can become more negative to the north and south of the jet center. The scatter diagram at day 9 shown in Fig. 7 was obtained from the weakly nonlinear model in LLW and is very similar to the theoretical result obtained by HMR by extending the Korteweg-de-Vries (KdV) solitary wave blocking model (Malguzzi and Malanotte-Rizzoli 1984). This model can be used to describe the jet intensification anomalies over the North Pacific. However, the HMR extended $\mathrm{KdV}$ model is only appropriate for the mature stage of an $\mathrm{NAO}^{+}$event. That is to say, the characteristics of the scatter diagrams in the early and decay stages of the intensified jet anomaly cannot be reflected by their model. Although the HMR model can capture some of the essential physics of jet intensification anomalies occurring in the Pacific and Atlantic sectors, it is the inability of the model to explain how the jet intensification anomalies ( $\mathrm{NAO}^{+}$events) in the Atlantic sector affect the blocking activity over the Europe. However, this issue can be investigated using the weakly nonlinear model proposed in LLW. Thus, it is natural to conclude that the weakly nonlinear model proposed in LLW is more appropriate for representing the influence of the NAO on European blockings.

Similarly, the dependence of the scatter diagram for the $\mathrm{NAO}^{+}$event at day 9 on the initial conditions is also examined, which is shown in Fig. 8 for two initial conditions of $B(x, 0)=B_{0}$ and $B(x, 0)=B_{0}$ $\operatorname{sech}\left(\sqrt{\delta /(2 \lambda)} B_{0} x\right)$ with $B_{0}=0.45$.

Note that the scatter diagram that exhibits an almost linear functional relationship does not depend strongly upon different choice of initial values. This indicates sufficiently that the $q_{P}-\psi_{P}$ plot of the $\mathrm{NAO}^{+}$event should essentially have an almost linear functional relationship. This allows us to speculate that the weakly nonlinear dynamics may be more important for the $\mathrm{NAO}^{+}$event. Even so, the $\mathrm{NAO}^{+}$event can be thought of as a free mode.

In previous studies, the free mode characteristics of split-jet anomalies can be approximately captured by the strongly nonlinear modon model of McWilliams (1980), but the free mode dynamics of the intensified jet anomalies can only be described by a weakly nonlinear model (HMR; Haines et al. 1993). In this paper, the weakly nonlinear model, proposed as a unified approach in LLW, is found to not only be able to capture the free mode characteristics of isolated split jet anomalies $\left(\mathrm{NAO}^{-}\right.$events), but also the free mode characteristics of intensified jet anomalies $\left(\mathrm{NAO}^{+}\right.$events).

In the diagnostic studies of observed blocking cases, an important feature of the $q(\psi)$ scatter diagram is the existence of a steepening prior to the full establishment of blocking (Butchart et al. 1989; Ek and Swaters 1994). However, the physical cause for this steepening on the scatter diagram is unclear, and this will be explored in the next section. In addition, some new features on the scatter diagrams of $\mathrm{NAO}^{-}$and $\mathrm{NAO}^{+}$events are also found here.

\section{Does the steepening of the scatter diagram exist for the two phases of NAO?}

It was found in Figs. 2, 5, and 7 that there was no steepening of the slope of the main straight line in the $q_{P}\left(\psi_{P}\right)$ scatter diagrams for two phases of the NAO events. However, Butchart et al. (1989) and Ek and Swaters (1994) found by calculating the $q(\psi)$ scatter diagrams of the five-day mean geostrophic streamfunc- 
(day 0 )

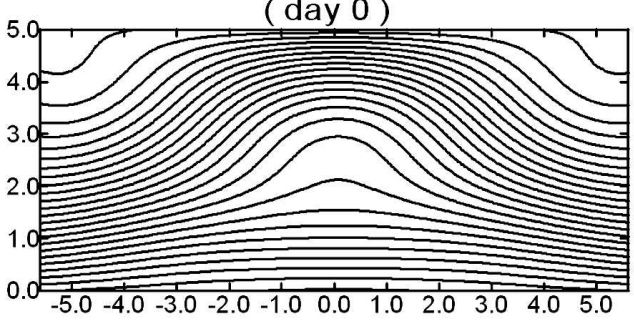

(day 6 )

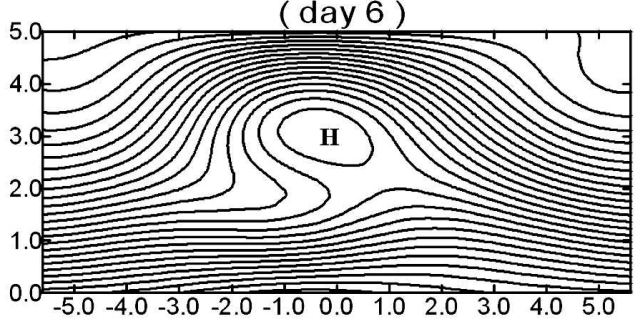

(day 12 )

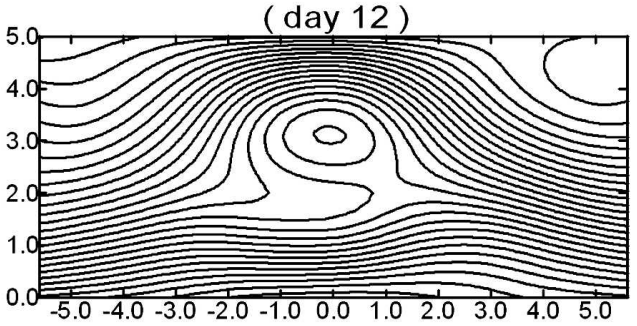

(day 18)

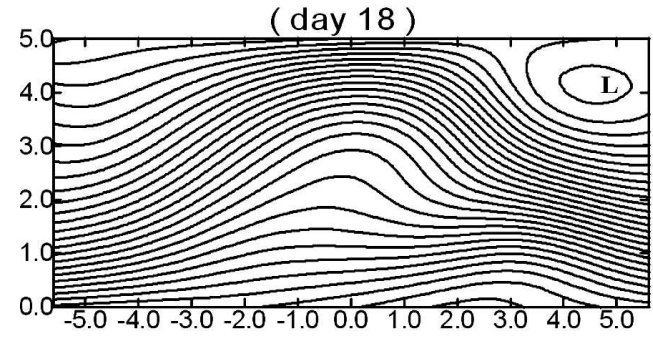

(day 3 )

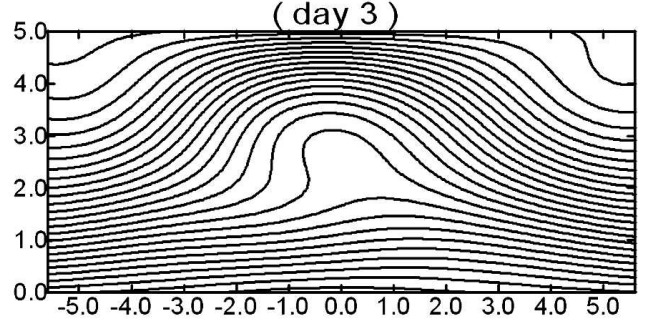

(day 9)

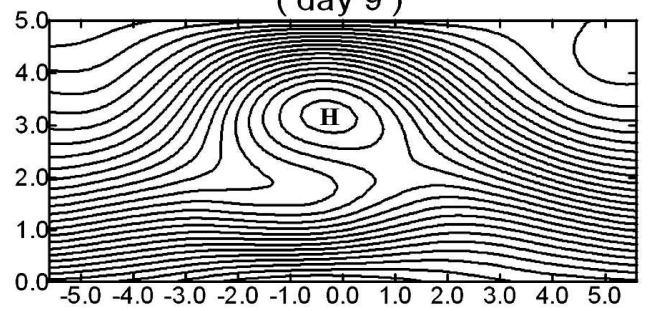

(day 15 )

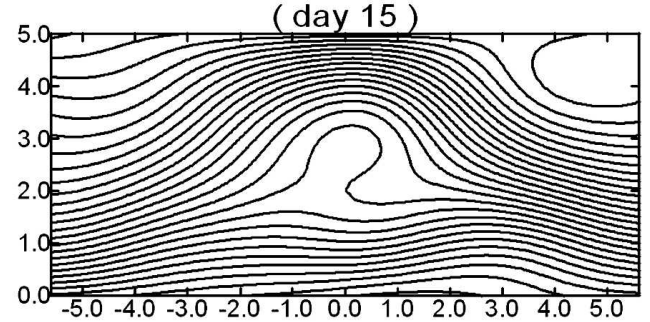

(day 21 )

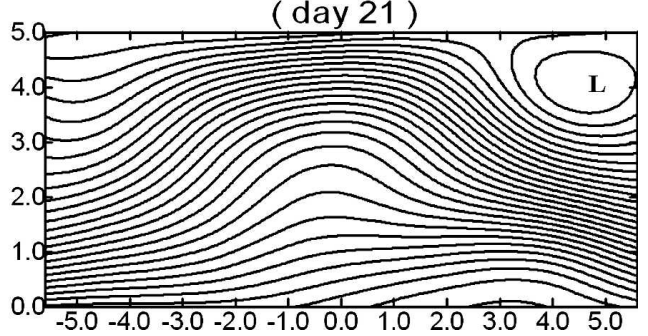

FIG. 4. Planetary-scale field of a negative-phase NAO event driven by both synoptic-scale eddies and large-scale LSC topography for an initial value of $B(x, 0)=B_{0} \cos (\varepsilon x), B_{0}=0.3$, and $\varepsilon=0.24$, and topographic parameter $h_{0}=0.5$ in the weakly nonlinear model proposed in Luo and Chen $(2006 \mathrm{~b} ; \mathrm{CI}=0.3)$.

tion against PV during blocking episodes that there is a steepening of the $q(\psi)$ scatter diagrams prior to blocking formation. Although their calculations involved actual blocking cases, observed $\mathrm{NAO}^{-}$events are also found to have such features in that $\mathrm{NAO}^{-}$events resemble blocking events in the Atlantic sector. This will be revealed by the composite field study of $\mathrm{NAO}^{-}$ events in the next section. However, a comparison with Figs. 2 and 5 indicates that the scatter diagrams obtained from the weakly nonlinear NAO model in LLW are not consistent with the scatter diagrams from data obtained by Ek and Swaters (1994). It is likely that the synoptic-scale eddies may play a role in causing this inconsistency. This is because the five-day mean fields cannot sufficiently filter out synoptic-scale eddies from the daily field. As pointed out by Butchart et al. (1989), a 5-day averaging had little effect on the overall spatial structure of the PV and streamfunction fields for observed blocking events except to reduce the amplitudes of the individual features. For this case, synoptic-scale eddies cannot almost be removed from the streamfunction field. Thus, their scatter diagrams actually include the role of synoptic-scale eddies. This point will be confirmed by the calculation of the scatter diagram of the streamfunction versus PV with and without the effect of synoptic- scale eddies in this section.

Figure 9 shows the scatter diagrams for the total streamfunction $\left(\psi_{T}\right)$ against its corresponding PV $\left(q_{T}\right)$ for $\mathrm{NAO}^{-}$and $\mathrm{NAO}^{+}$events shown in LLW (their Figs. $3 c$ and $5 c$ ). It can be seen from Fig. 9 that as 

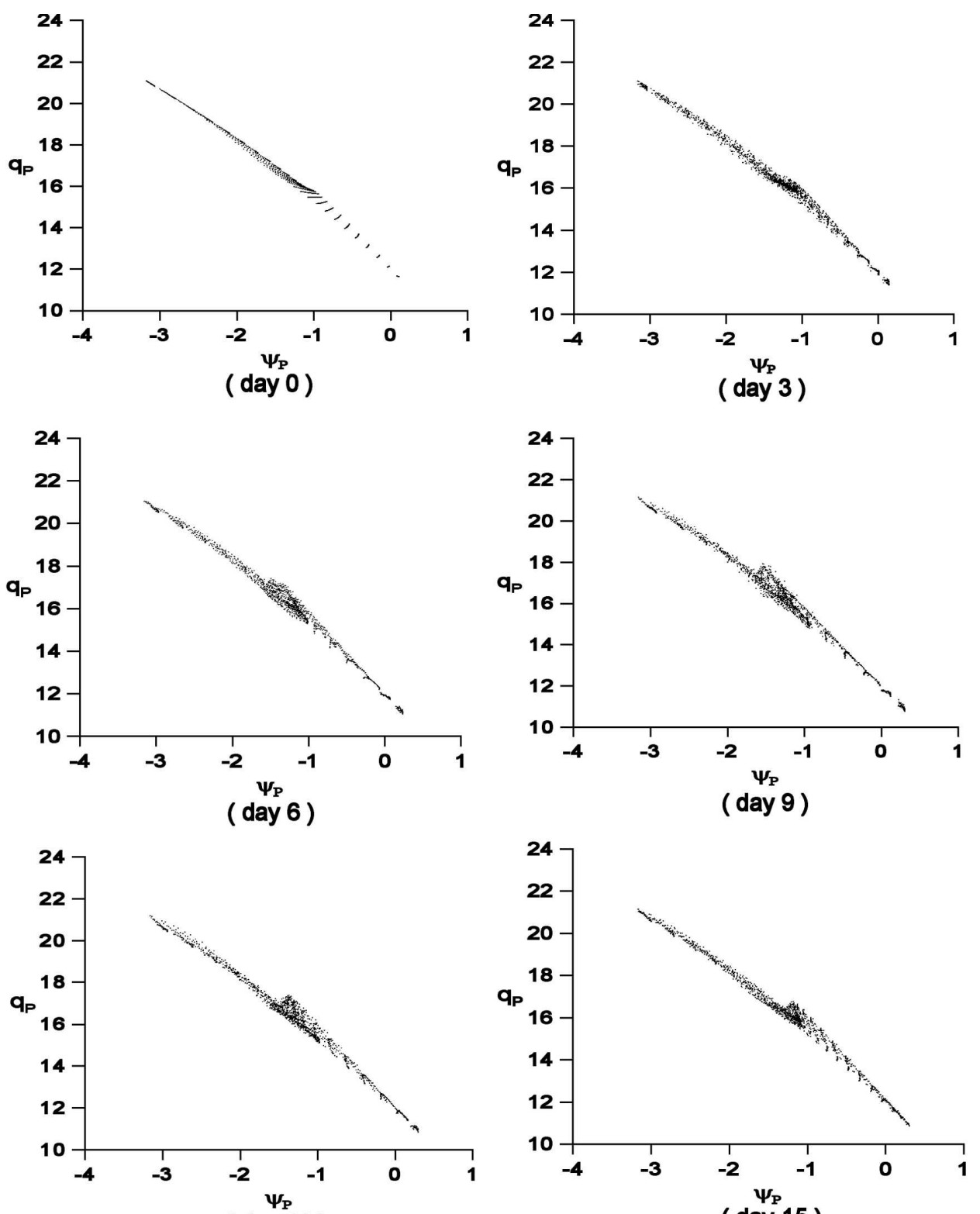

( day 12 )

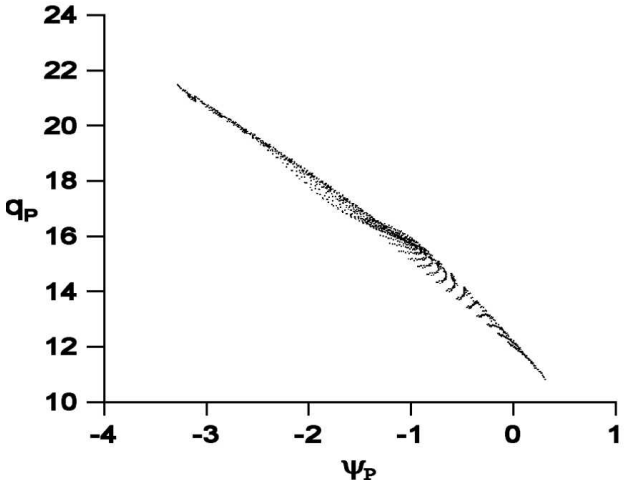

(day 18)

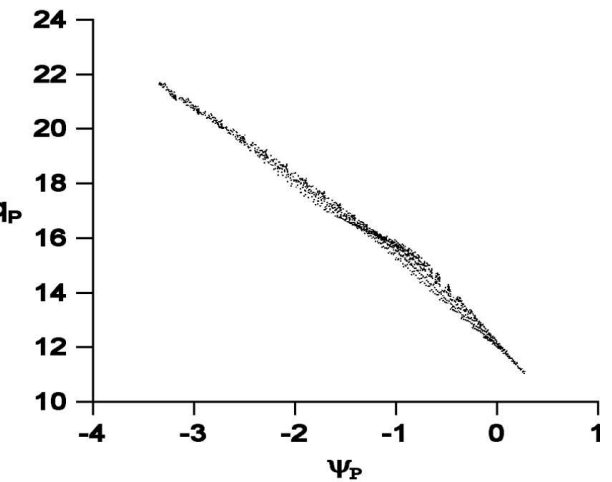

( day 21 )

FIG. 5. Scatter diagrams of $\psi_{P}$ vs $q_{P}$ for the life cycle of the negative-phase NAO event as shown in Fig. 4. 
(day 0 )

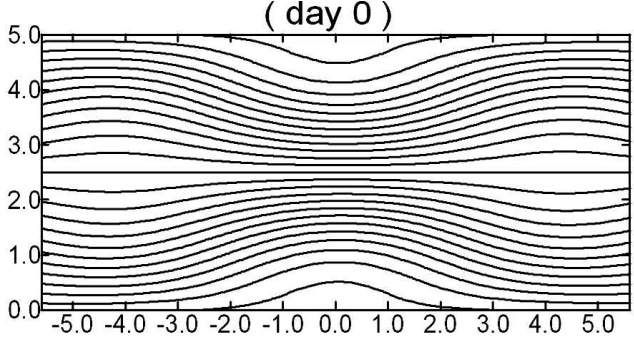

(day 6 )

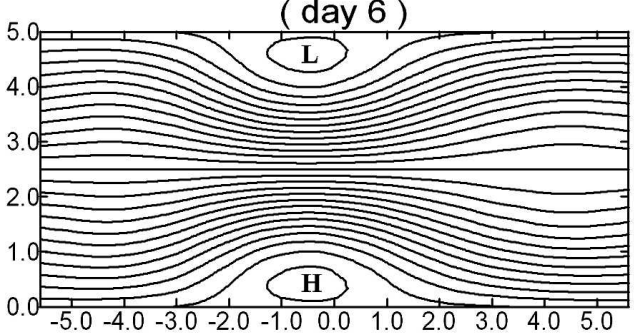

( day 12)

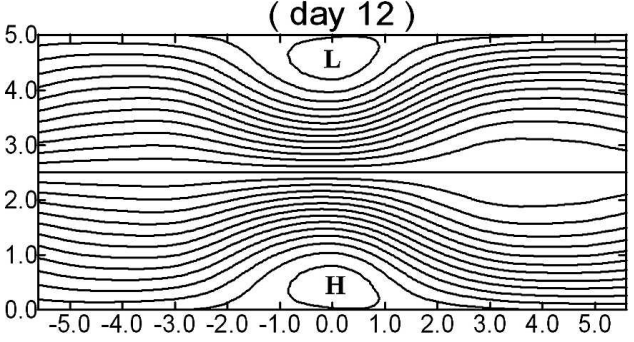

( day 18 )

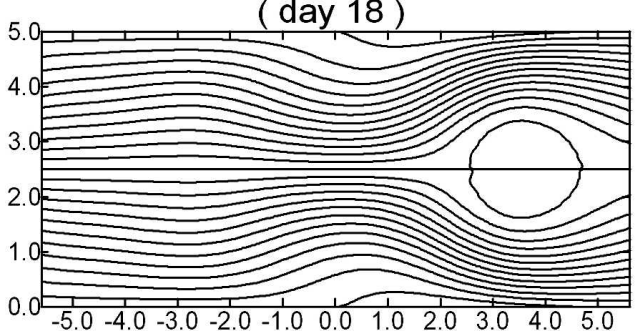

(day 3 )

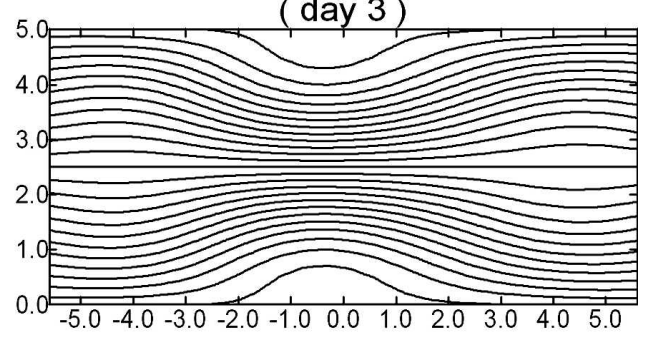

( day 9 )

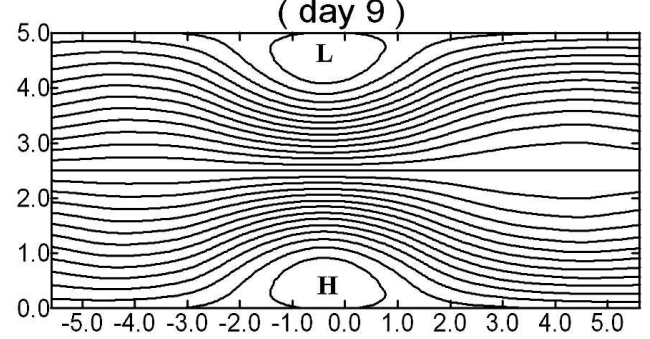

(day 15 )

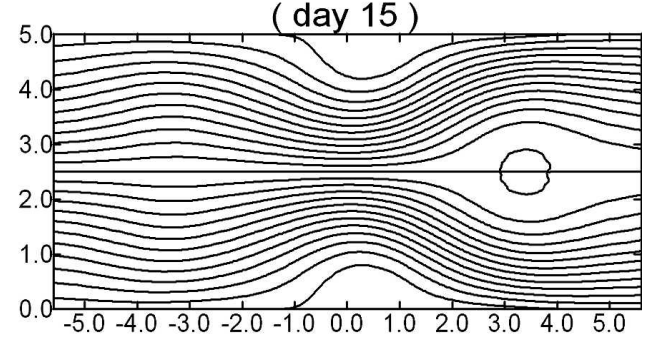

( day 21 )

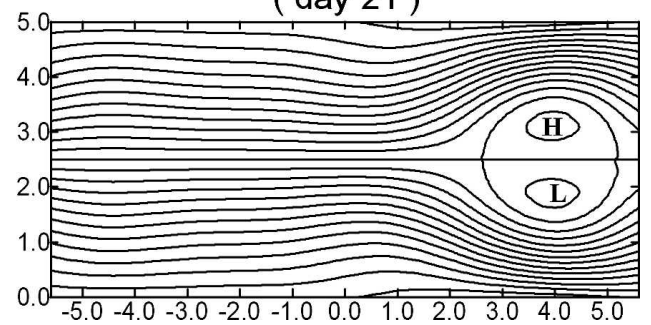

FIG. 6. Planetary-scale fields for the life cycle of a positive-phase NAO event, in which the CI is 0.3 (from LLW).

synoptic-scale eddies are included in the data used, the $q_{T}\left(\psi_{T}\right)$ scatter diagram for the $\mathrm{NAO}^{-}$event tends to steepen during the intensification phase. This tendency reverses, however, during the decay phase (Fig. 9a), which is unlike the characteristics of the $q_{P}\left(\psi_{P}\right)$ scatter diagram found in Fig. 2. Also, it is interesting to see that the $q_{T}\left(\psi_{T}\right)$ scatter diagram for the $\mathrm{NAO}^{+}$event shows a trend toward a shoaling during the intensification phase, while it steepened during the decay phase (Fig. 9b). Thus, a comparison with Figs. 2 and 7 indicates that the steepening and shoaling of the $q_{T}\left(\psi_{T}\right)$ scatter diagram of the streamfunction versus PV for the two phases of the NAO can be attributed to the effect of synoptic-scale eddies. In this process, the northward (southward) shift of synoptic-scale anticyclones (cyclones) associated with the NAO growth results in the steepening in the $q_{T}\left(\psi_{T}\right)$ scatter diagram. But a reverse trend of synoptic-scale eddies associated with the NAO decay tends to induce the shoaling in the $q_{T}\left(\psi_{T}\right)$ scatter diagram. Ek and Swaters (1994) suggested that the steepening of the scatter diagram prior to the formation of the block is associated with an intense cyclonic disturbance (Arctic disturbance), this was also noted by Butchart et al. (1989). Unfortunately, the shoaling of the slope of the scatter diagrams during the decay phase, as shown in Fig. 9a, is not noted in their diagnostic studies. The diagnostic study in section 5 will indicate this point. 

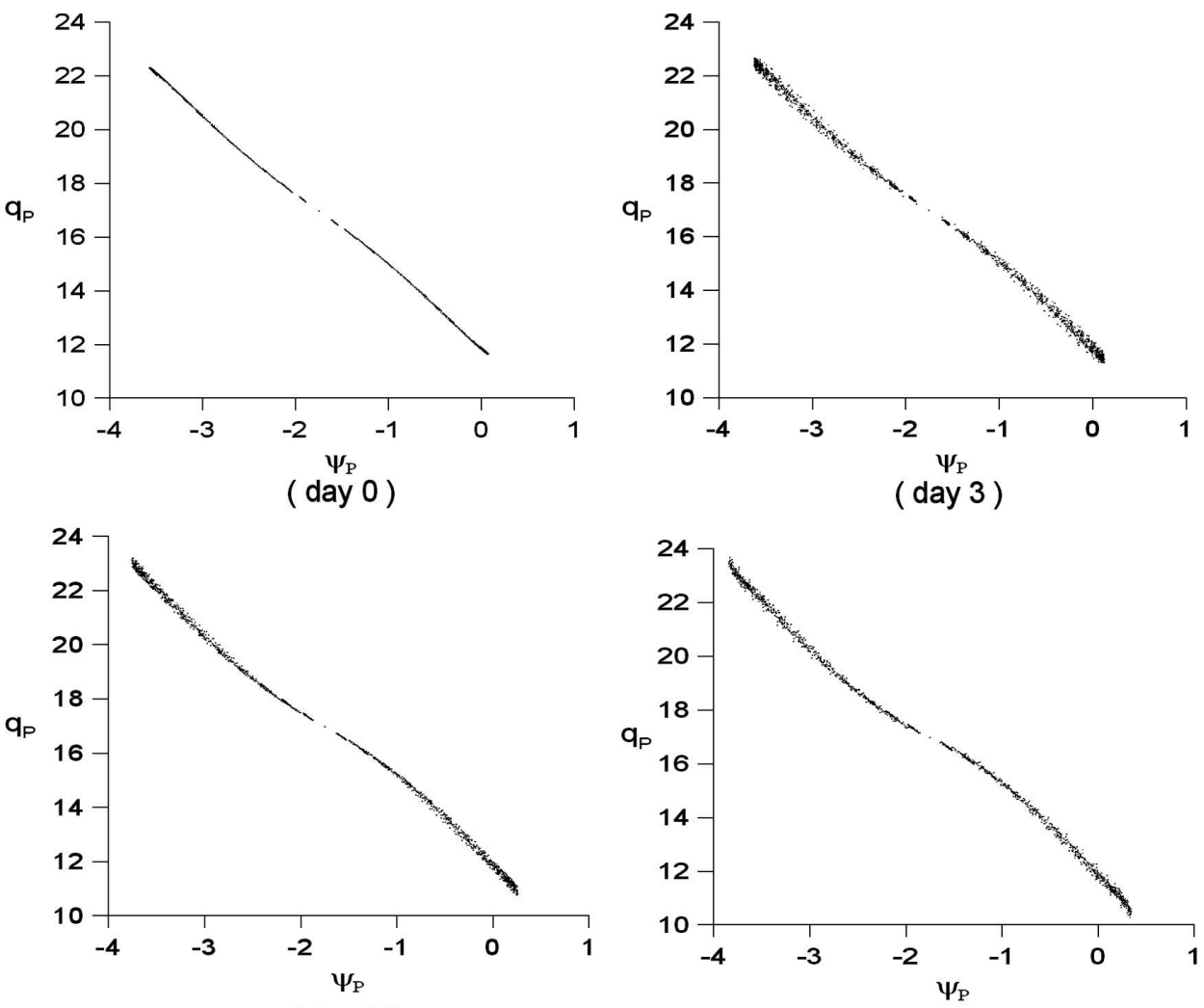

( day 6 )
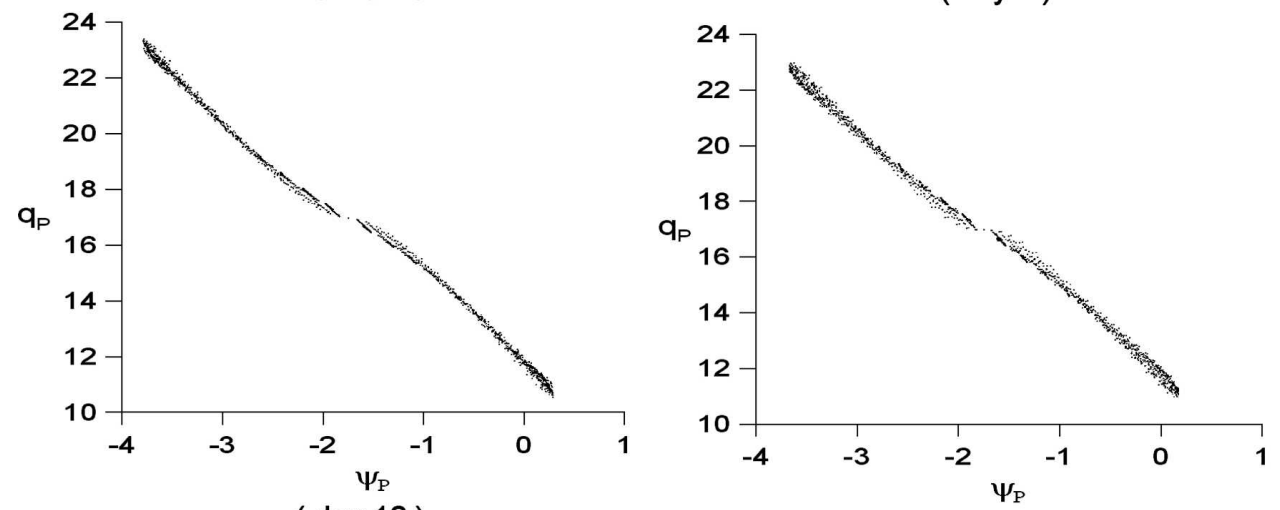

( day 12 )

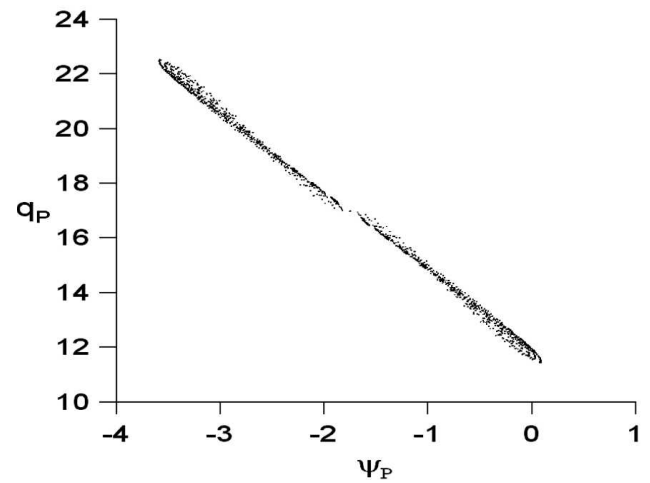

( day 18 )

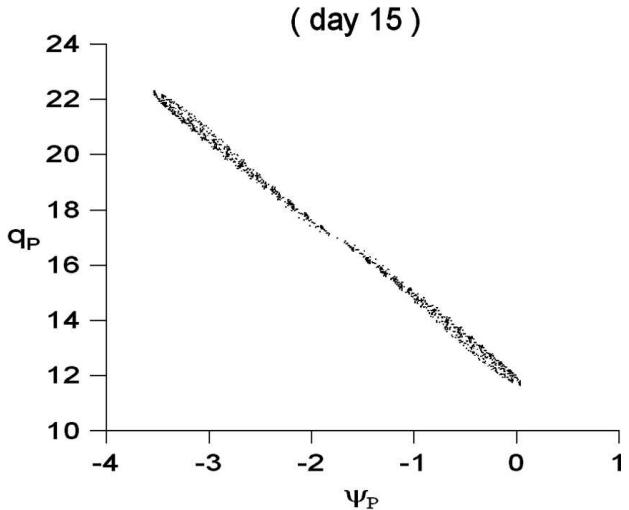

( day 21 )

FIG. 7. Scatter diagrams of $\psi_{P}$ vs $q_{P}$ for the life cycle of the positive-phase NAO event as shown in Fig. 6. 
(a)

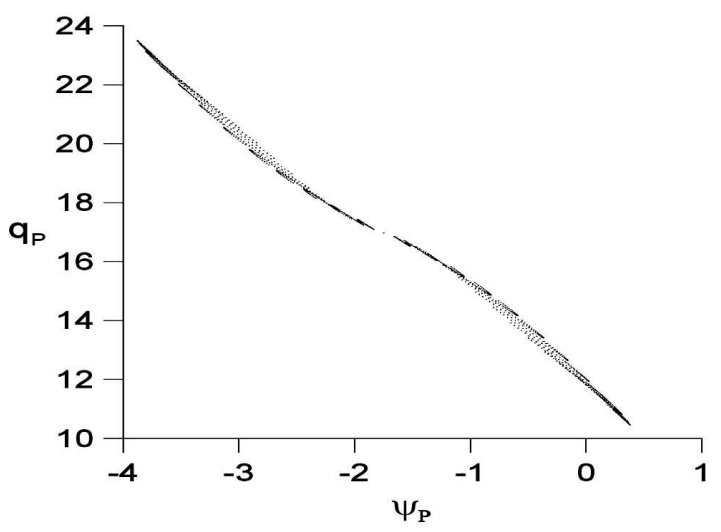

(b)

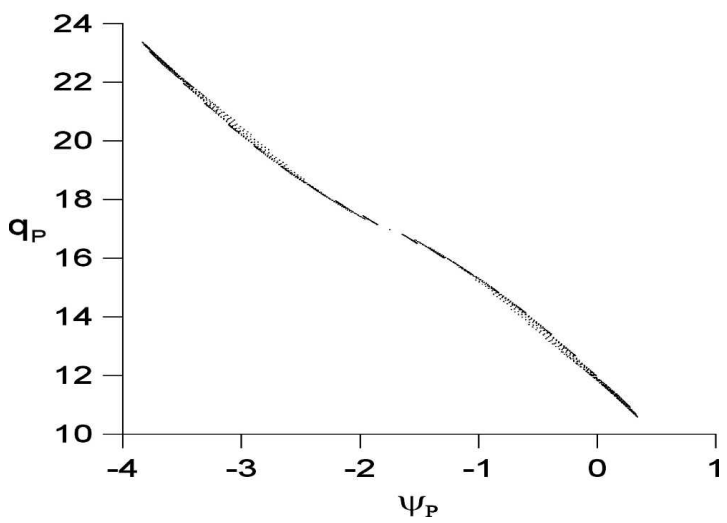

FIG. 8. Scatter diagrams of $\psi_{P}$ vs $q_{P}$ of a positive-phase NAO event at day 9 for two different initial values: (a) initial value of $B(x, 0)=B_{0}$ for $B_{0}=0.45$ and (b) initial value of $B(x, 0)=B_{0}$

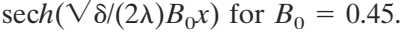

In addition, we can see in Fig. 9a that the $q_{T}\left(\psi_{T}\right)$ scatter diagram of the $\mathrm{NAO}^{-}$event (blocking) shows a linear multivalued functional relationship, which is likely to be modonlike, but the $\mathrm{NAO}^{+}$(persistent intensified jet) scatter diagram is not similar (Fig. 9b). Thus, it is conjectured that two approximate straight lines in the scatter diagram of the $\mathrm{NAO}^{-}$event (blocking event) may also be associated with upstream synoptic-scale eddies. But for the $\mathrm{NAO}^{+}$phase, the $q_{T}\left(\psi_{T}\right)$ scatter diagrams do not show such a characteristic. To further confirm the above ideas, a diagnostic composite study of observed NAO events and its diagnostic study are performed based upon the daily NAO index by Benedict et al. (2004).

\section{Characteristics of the scatter diagrams in the composite fields of negative- and positive-phase NAO events and free modes}

Before evaluating the relevance of the weakly nonlinear model in LLW to NAO events, a sample of 10 positive and 20 negative NAO events during the period spanning the years 1958-97 for the months of December-February (DJF) was chosen, Following Benedict et al. (2004), we used the daily NAO index time series to select the NAO events used for a composite (see Fig. 1 of Benedict et al., 2004). The data used for our composite are the daily (0000 UTC) National Centers for Environmental Prediction-National Center for Atmospheric Research (NCEP-NCAR) reanalysis height at 300-mb level and multilevel (500, 400, 300, 250, 200, and $150 \mathrm{mb}$ ) temperature data on a latitude-longitude grid with $2.5^{\circ}$ interval.

As in Benedict et al. (2004), a series of $\mathrm{NAO}^{-}$and $\mathrm{NAO}^{+}$events are defined based on the first rotated principal component analysis (RPCA) of the $300-\mathrm{mb}$ geopotential height field. An event is specified as a string of four or more consecutive days in which the NAO index is greater than 1.33 standard deviations. The zero-lag day that is the base point of an event is defined as the day on which the index is at its largest value. Using unfiltered $300-\mathrm{mb}$ geopotential height fields, we can produce two sets of composite fields aligned to the zero-lag day, one for the selected 10 negative-phase cases and the other for the 20 positivephase cases. The composite of the NAO events should, to a large extent, filter out the synoptic-scale eddies, which excite the NAO events. In this case, the composite field of the NAO events can represent the planetaryscale part of the NAO flow.

Figure 10 shows the composite geopotential height fields at the 300-mb level for both $\mathrm{NAO}^{-}$and $\mathrm{NAO}^{+}$ events from Benedict et al. (2004). It can be shown in Fig. 10a that the composite field of $\mathrm{NAO}^{-}$events at the $300-\mathrm{mb}$ level represents the life cycle of an Omega type block. Figure 10a also bears a striking resemblance to Fig. 4, but the composite field of $\mathrm{NAO}^{+}$events exhibits a low-over-high dipole structure, the intensified jet anomaly (Fig. 10b), which looks similar to Fig. 6. The zero-lag day in the two figures corresponds to the strongest stage of the composite NAO event. However, it must be pointed out that the decay of the omega type blocking structure in Fig. 10a is a rapid process, which may be related to the baroclinicity of the background flow (Luo 2005). To further evaluate the relevance of the weakly nonlinear model in LLW to observed NAO events, the scatter diagrams of the streamfunction versus PV for the composite fields of the NAO events for two phases are calculated using the composite geostrophic streamfunction. Throughout this calculation, the box plotted in Fig. 10 is subjectively chosen to surround a region used to compute the scatter diagrams of the streamfunction versus PV for the NAO events.

The geostrophic streamfunction was calculated at 
(a)

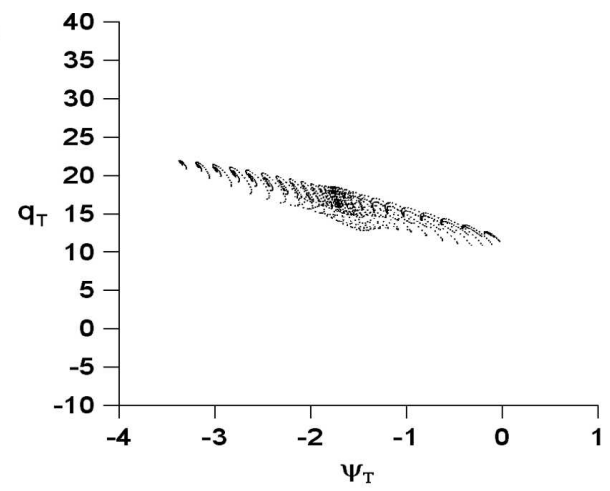

( day 0 )

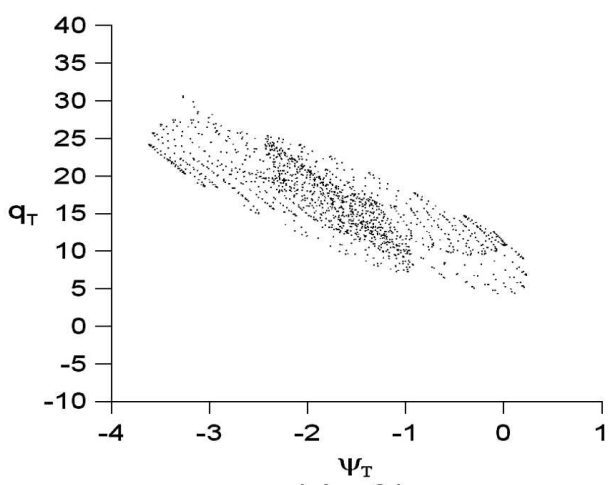

( day 6 )

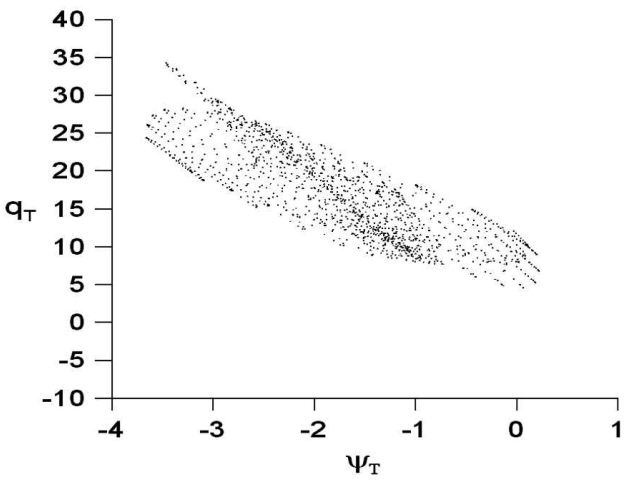

( day 12 )

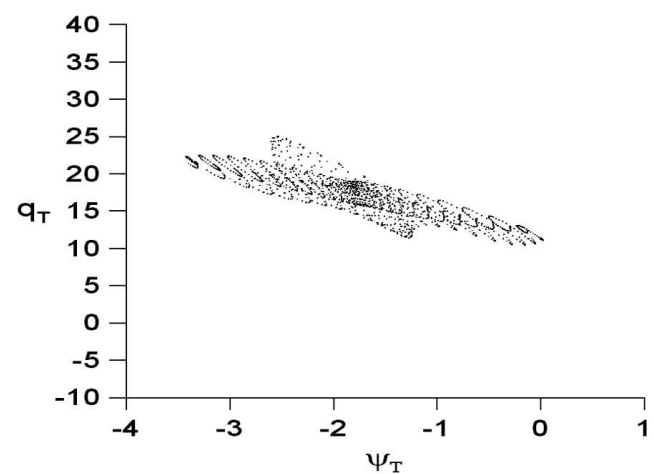

( day 18 )

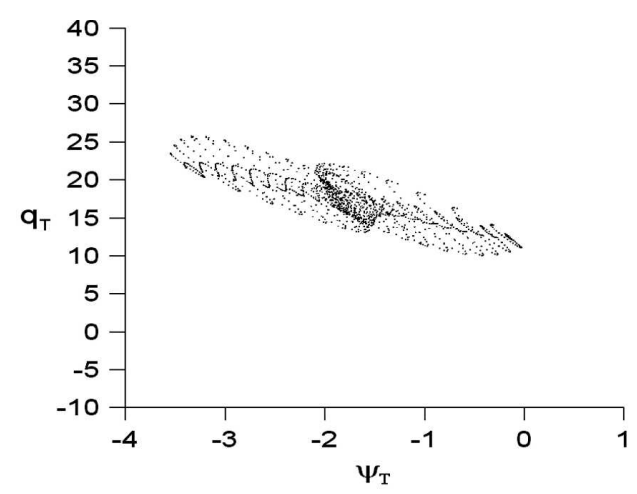

( day 3 )

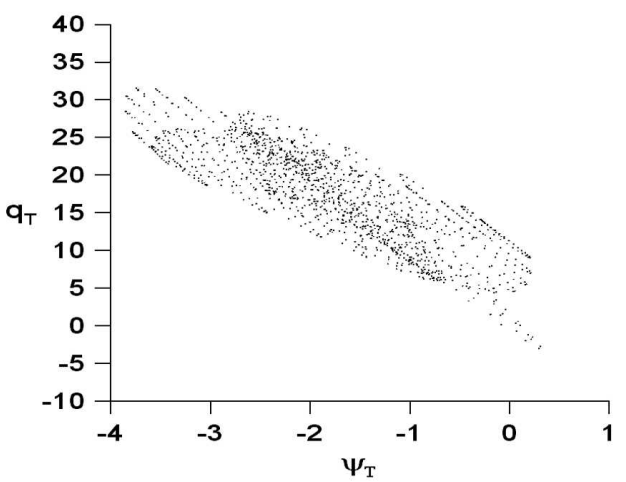

( day 9 )

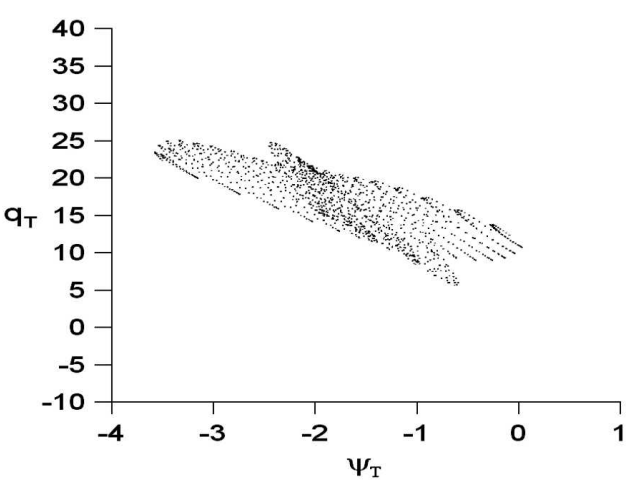

( day 15 )

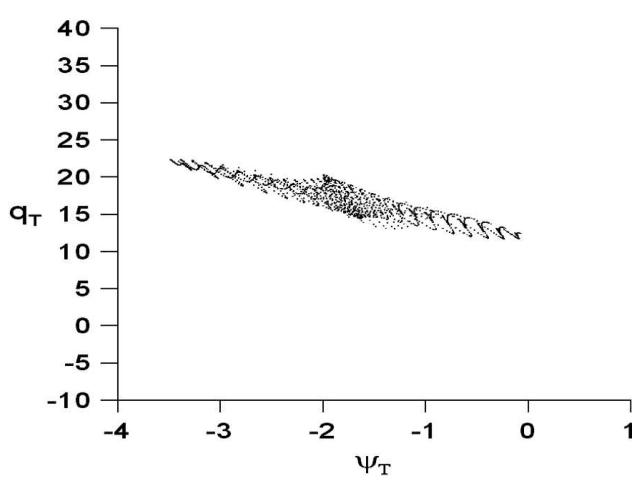

( day 21 )

FIG. 9. Scatter diagrams of the total streamfunction $\left(\psi_{T}\right)$ against its corresponding PV $\left(q_{T}\right)$ for negative- and positive-phase NAO events in Figs. 1 and 6, respectively, as shown in LLW (their Figs. 3c and 5c): (a) negative phase and (b) positive phase. 
(b)

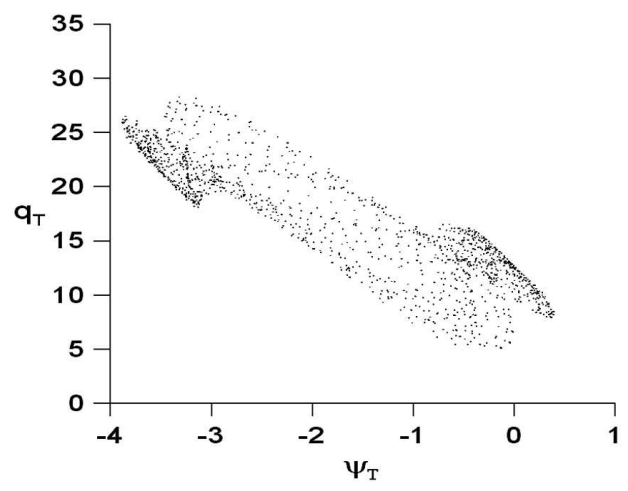

(day 0 )

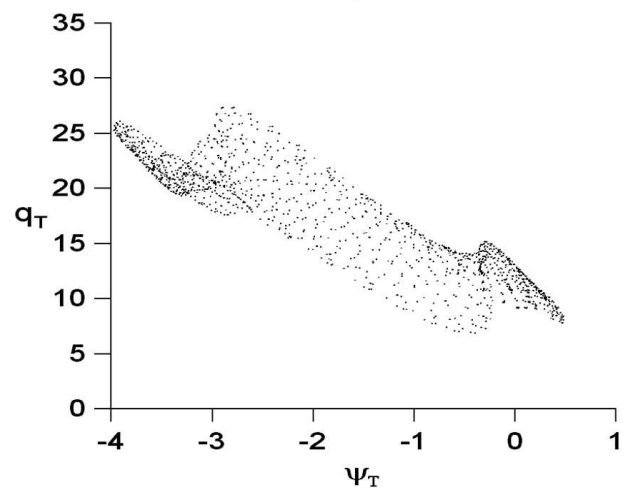

( day 6 )

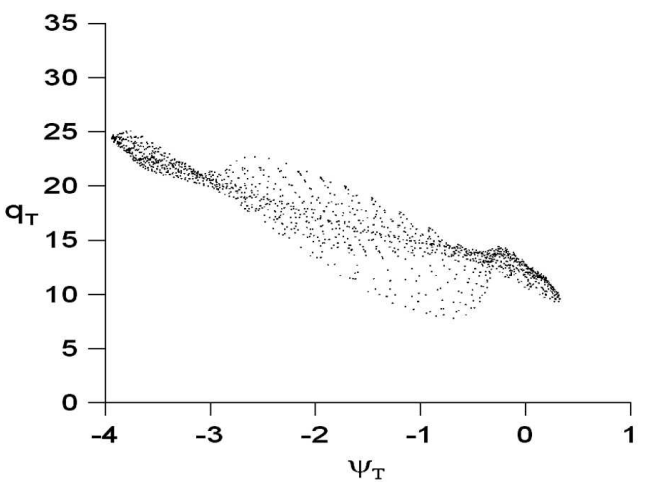

(day 12 )

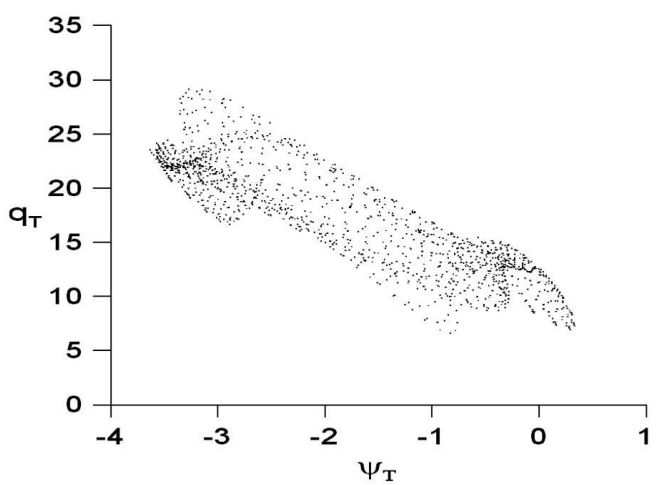

(day 18)

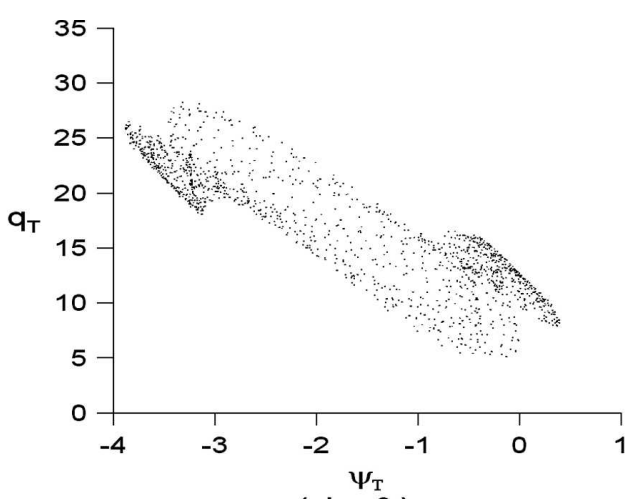

( day 3 )

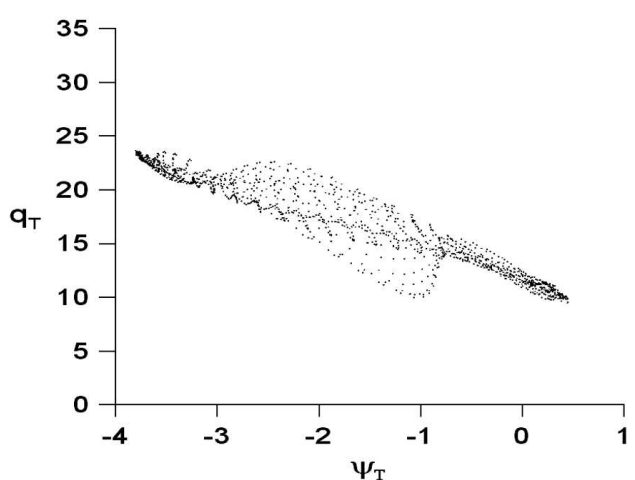

(day 9 )

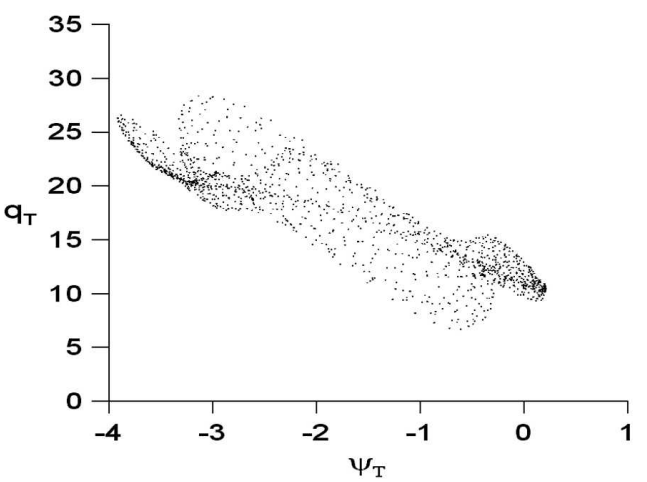

( day 15 )

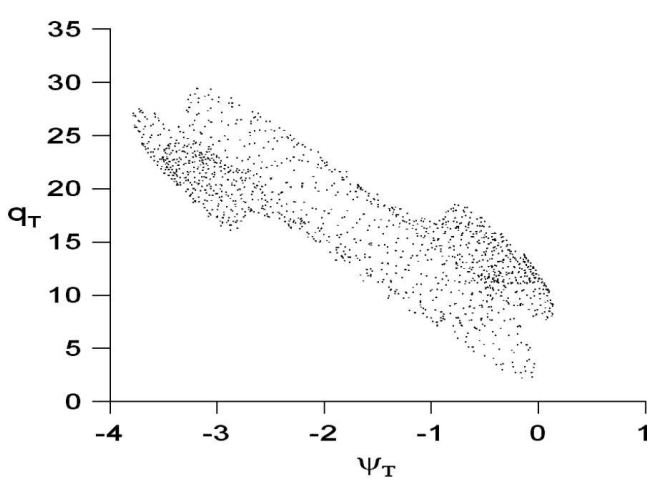

( day 21 )

FIG. 9. (Continued) 
(a)
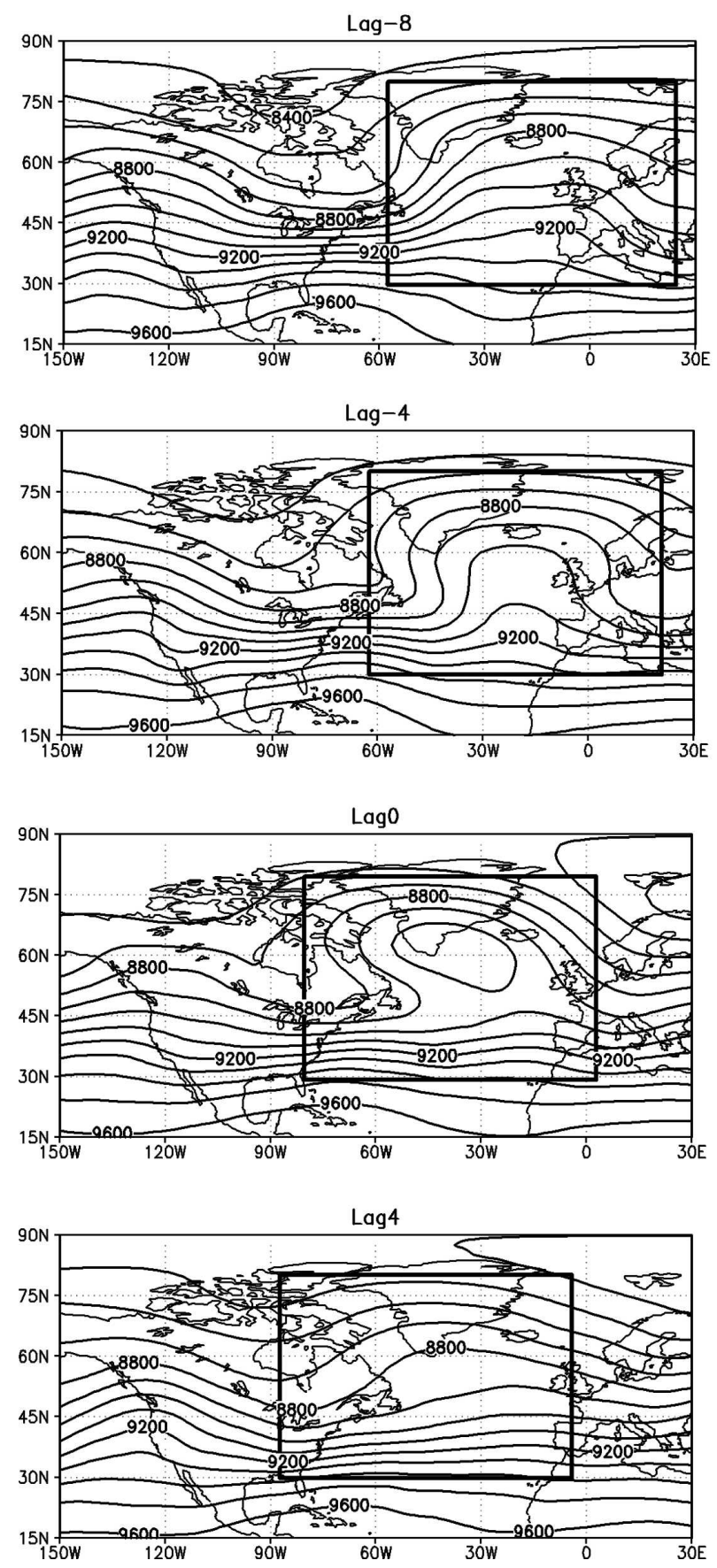

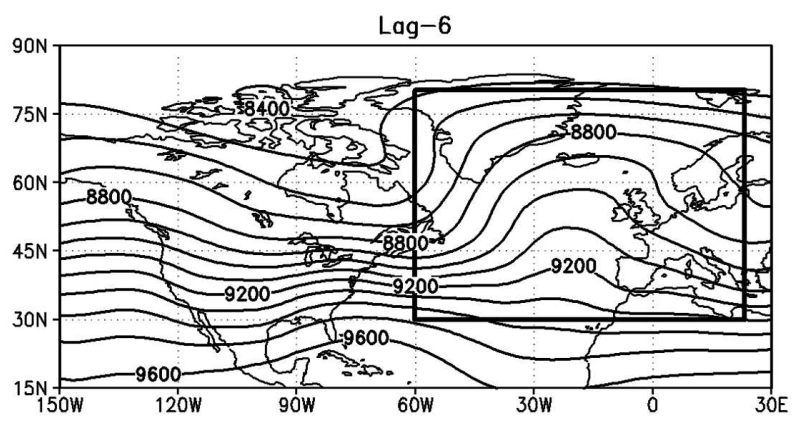

$\operatorname{Lag}-2$
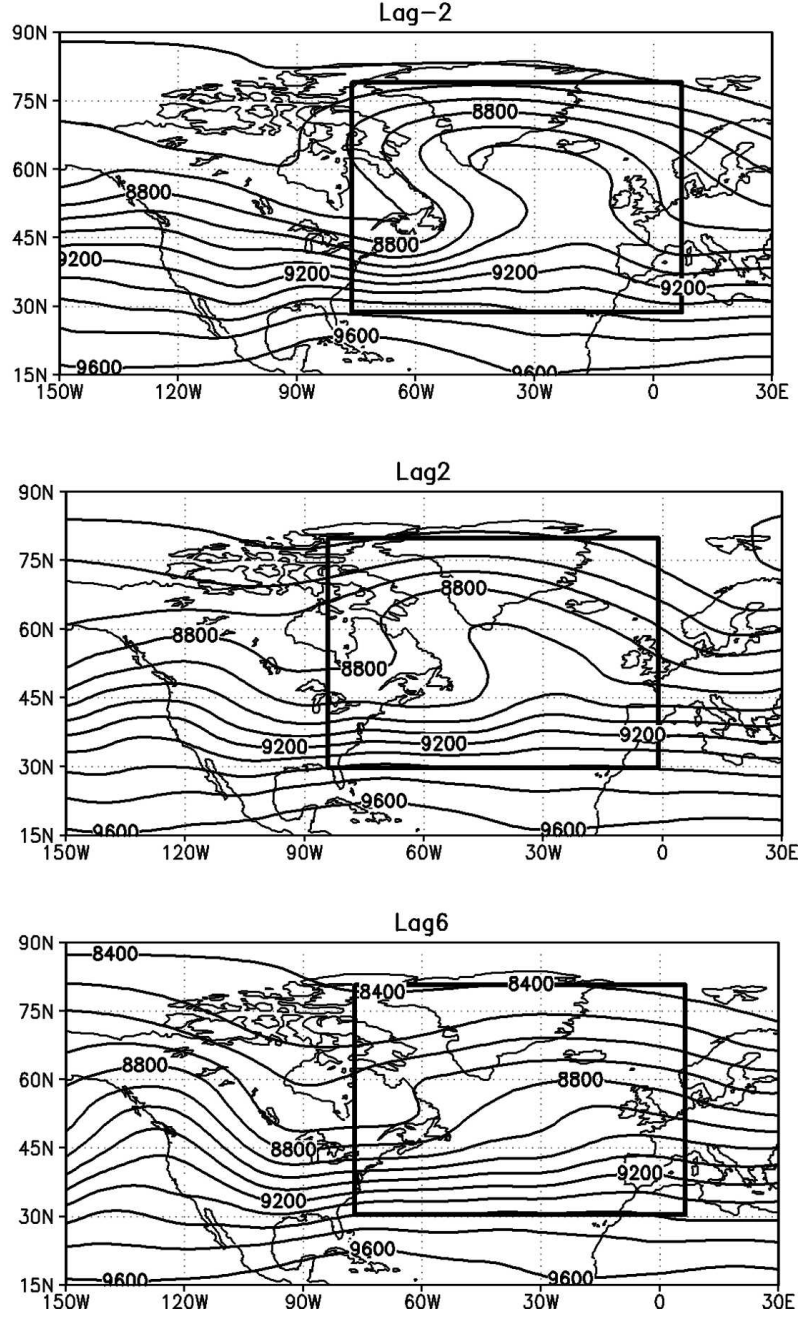

FIG. 10. Composite geopotential height fields at the 300-mb level of the NAO events for negative and positive phases according to the negative- and positive-phase NAO events presented by Benedict et al. (2004): (a) negative-phase field and (b) positive-phase field. The box in the two figures covering the main region of the NAO event is used to compute the scatterplots of the streamfunction against the corresponding PV.

each grid point $(i, j)$ as $\psi_{i, j}=g Z_{i, j} / f_{0}$, where $g$ is the gravitational acceleration, $f_{0}$ the Coriolis parameter at $55^{\circ} \mathrm{N}$, and $Z_{i, j}$ the geopotential height at the $300-\mathrm{mb}$ level. In this section, $Z_{i, j}$ is the composite geopotential height at the 300-mb surface, but is chosen to be an instantaneous $300-\mathrm{mb}$ geopotential height in the next section.

Similar to Butchart et al. (1989), the PV is calculated 
(b)
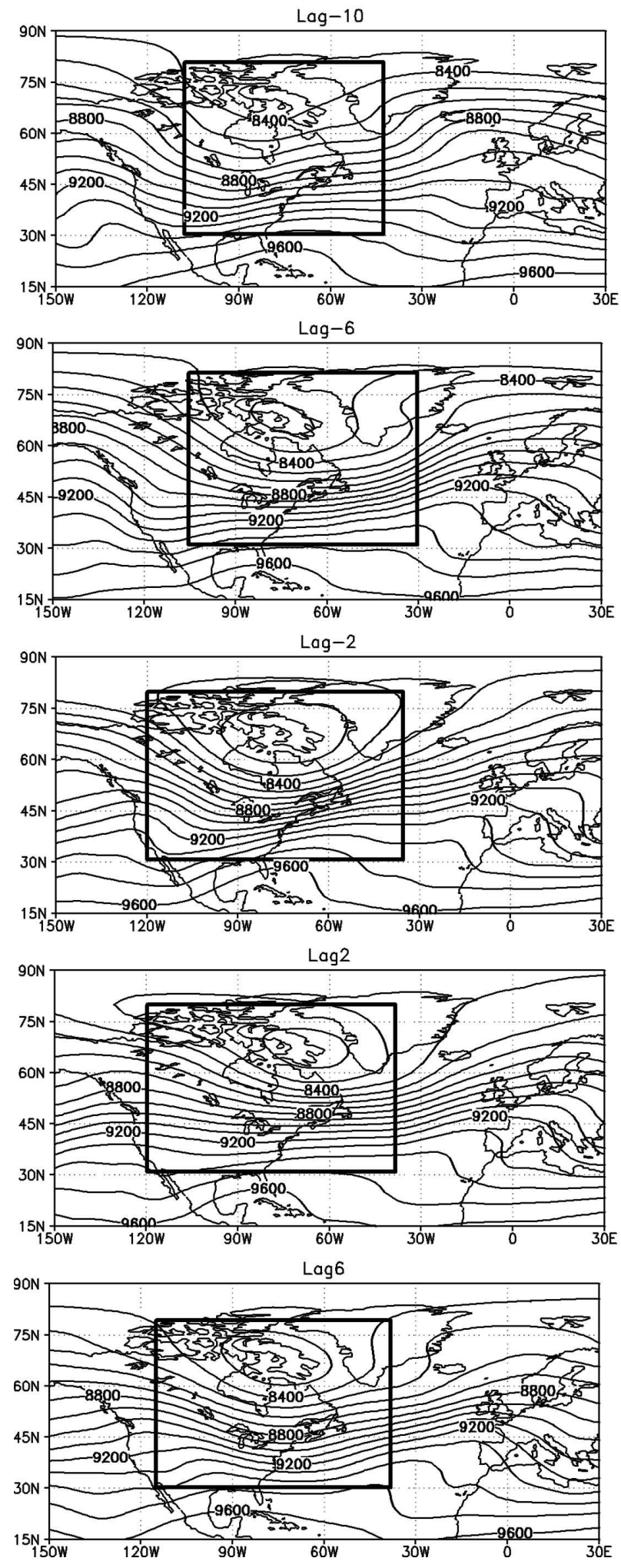
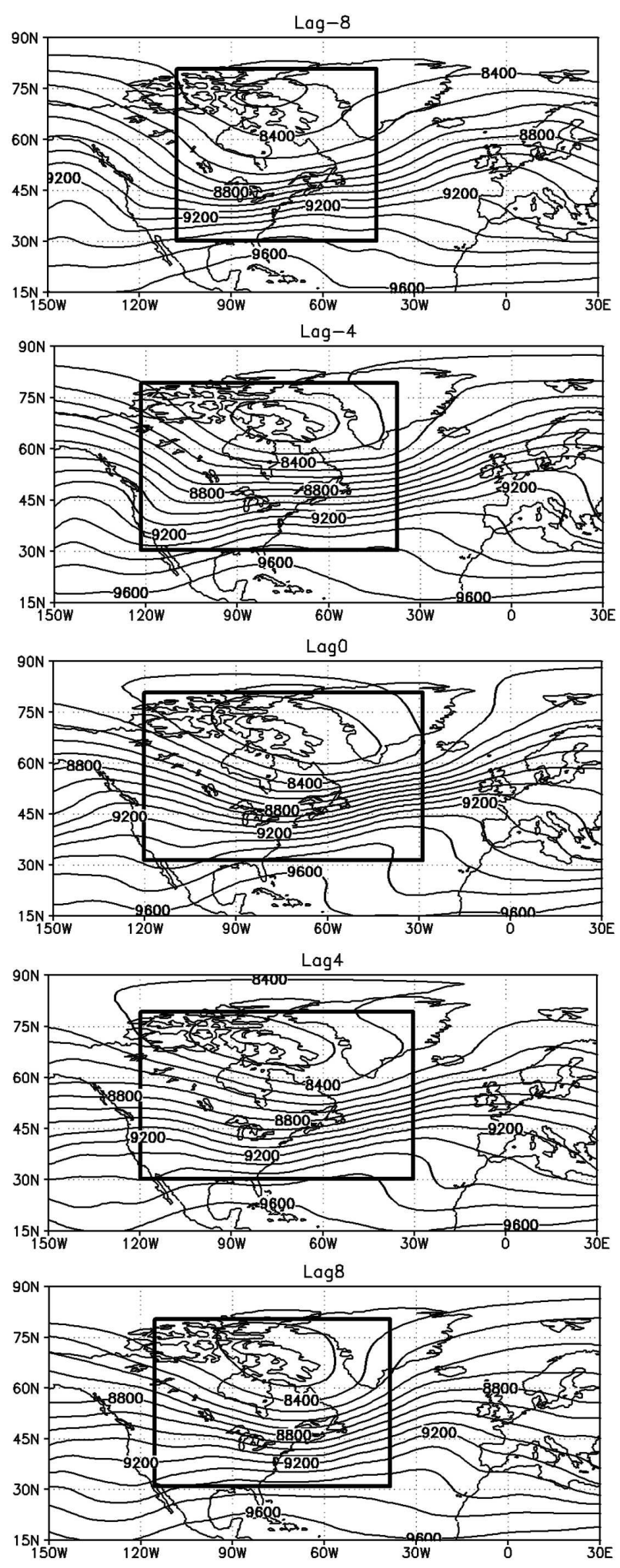

FIG. 10. (Continued) 
(a)
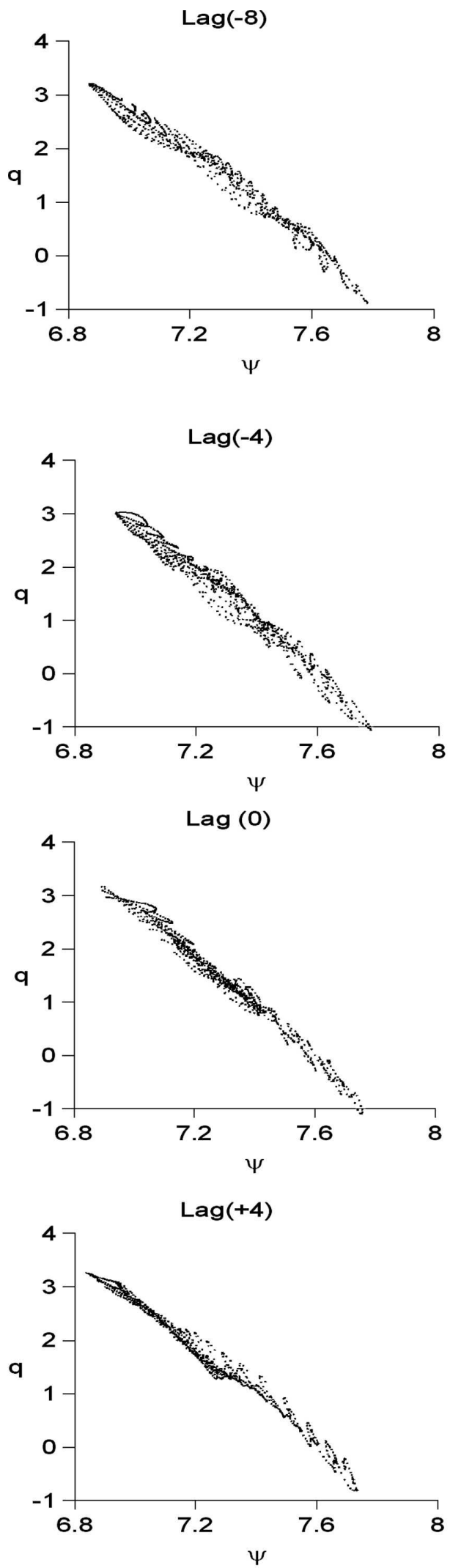
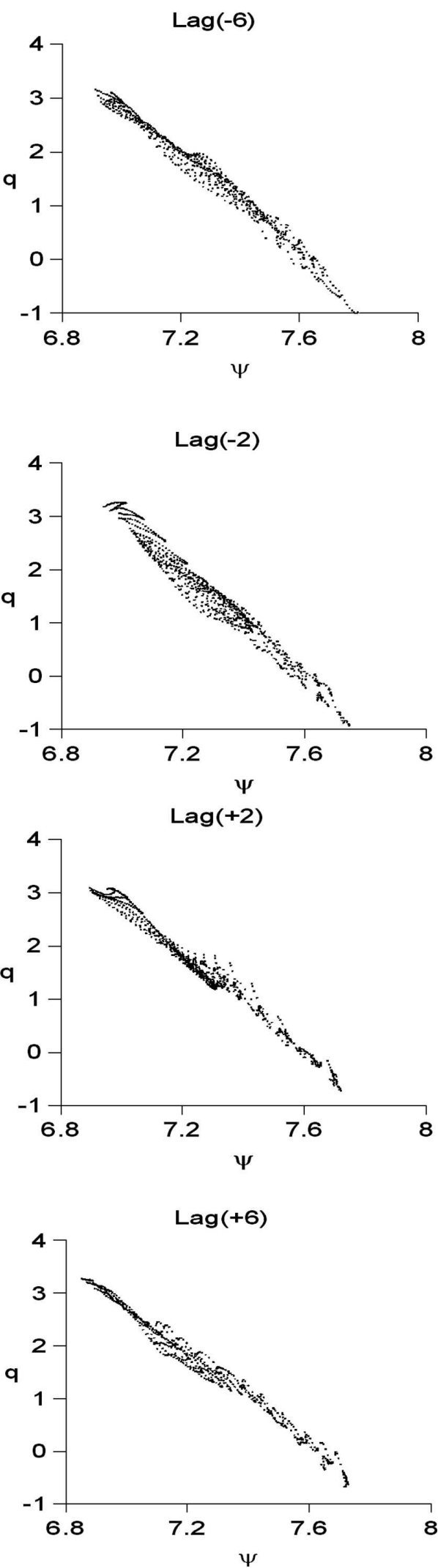

FIG. 11. Scatter diagrams of the streamfunction $\left(10^{8} \mathrm{~m}^{2} \mathrm{~s}^{-1}\right)$ against its corresponding PV $\left(10^{-4} \mathrm{~s}^{-1}\right)$ for the composite fields of negative- and positive-phase NAO events shown in Fig. 10: (a) negative phase and (b) positive phase 
(b)
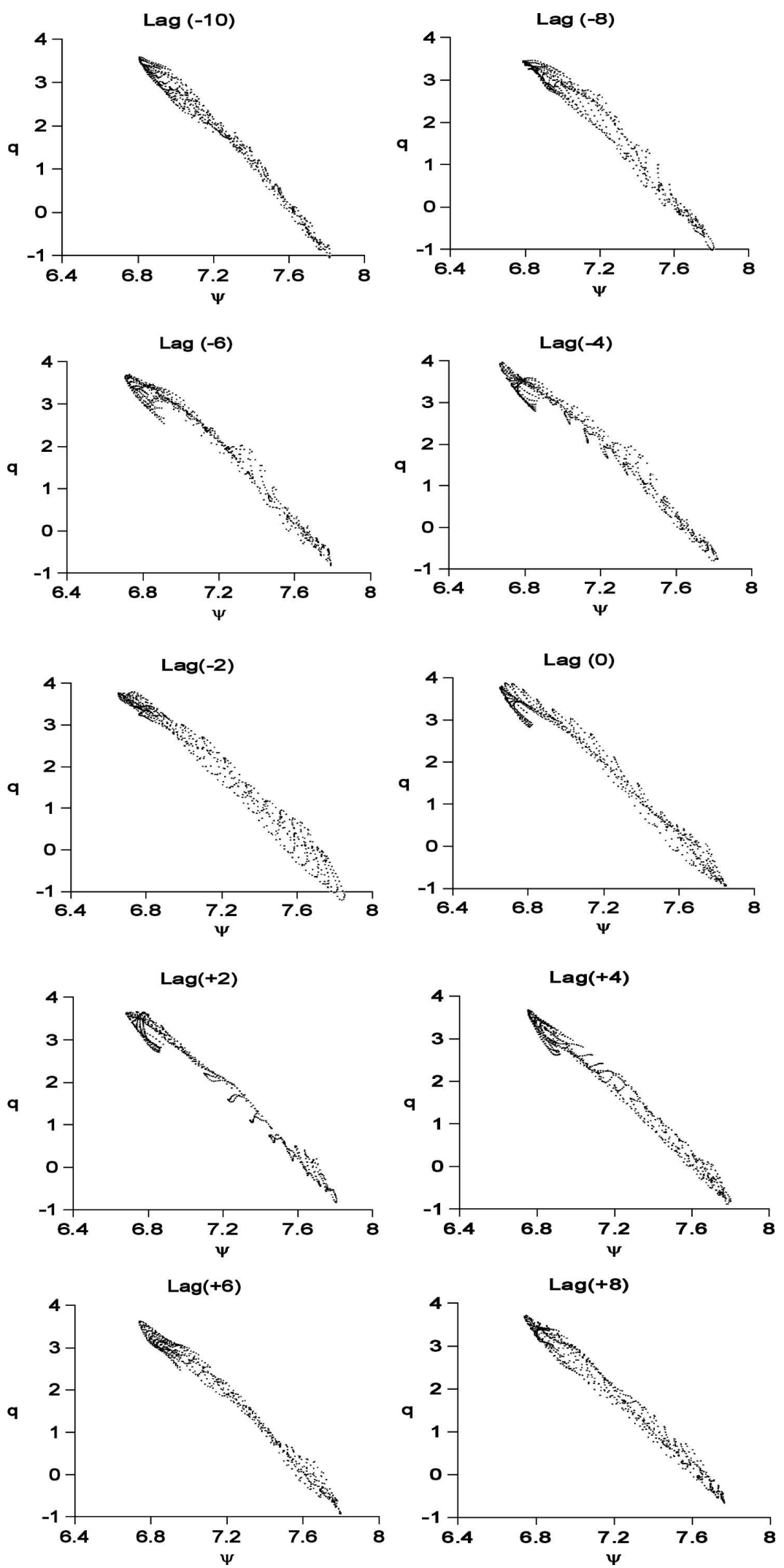

FIG. 11. (Continued) 
using the NCEP-NCAR reanalysis data in the expression

$$
q=f+\nabla^{2} \psi+f_{0} \frac{\partial}{\partial p}\left(\frac{\theta^{\prime}}{d \theta_{\mathrm{ref}} / d p}\right),
$$

where $\theta_{\text {ref }}$ is a reference potential temperature calculated as the area mean of a potential temperature at the $300-\mathrm{mb}$ level to the north of $22^{\circ} \mathrm{N}$, and $\theta^{\prime}$ is the deviation from the reference potential temperature.

Figure 11 shows the scatter diagrams of the streamfunction against $\mathrm{PV}$ for the composite fields of $\mathrm{NAO}^{-}$ and $\mathrm{NAO}^{+}$events shown in Fig. 10. It is found in Fig. 11a that for the $\mathrm{NAO}^{-}$event the scatter diagram of the streamfunction against PV exhibits a linear multivalued functional relationship in a narrow region, which seems to become more evident after the zero-lag day. It is also interesting to note that the scatterplots from lag 0 to lag +5 look similar to the scatter diagrams from day 9 to 15 in Fig. 5. This similarity may be due to a fact that the discrepancy between the two $\mathrm{NAO}^{-}$events shown in Figs. 11a and 5, respectively, is rather small. On the other hand, we see in Fig. 11a that the scatter diagram of the composite $\mathrm{NAO}^{-}$event does not show any steepening and shoaling during much of its evolution. This further supports our theoretical result in Fig. 5 where no steepening and shoaling of the $q_{P}\left(\psi_{P}\right)$ scatter diagram is observed. This shows that the weakly nonlinear model in LLW can capture the free mode characteristics of the composite $\mathrm{NAO}^{-}$event.

Also, it can be noted in Fig. 11b that the scatter diagram of the streamfunction versus PV for the composite $\mathrm{NAO}^{+}$event exhibits a linear single-valued functional relationship, and its slope seems unchanged in time except at lag $(-10)$. These features are in agreement with the theoretical finding in Fig. 7. In other words, in a pure planetary-scale field no shoaling and steepening of the scatter diagram can be detected for the $\mathrm{NAO}^{+}$event.

Therefore, the above diagnostic results strongly suggest that in a planetary-scale field there is no steepening and shoaling of the $q_{P}\left(\psi_{P}\right)$ scatter diagram for both phases of the NAO. As we will demonstrate in the next section, the steepening and shoaling of the scatter diagram of the streamfunction versus its corresponding $\mathrm{PV}$ for $\mathrm{NAO}^{-}$and $\mathrm{NAO}^{+}$events are likely to be associated with synoptic-scale eddies.

\section{Diagnostic studies of negative- and positive-phase NAO cases}

In this section, we will present the calculation of the scatter diagram of the streamfunction versus its corresponding PV from unfiltered geopotential height fields for two observed $\mathrm{NAO}^{-}$and $\mathrm{NAO}^{+}$cases. This calculation will confirm the important result from the above sections that the steepening and shoaling of the scatter diagram of the NAO event for its two phases are attributed to the effect of synoptic-scale eddies.

Figure 12 shows the geopotential height fields at 300 $\mathrm{mb}$ for two observed NAO events that represent negative and positive phases occurring in the Atlantic sector during the periods from 7 to 22 February 1969, and from 19 December 1989 to 7 January 1990.

It was found in Fig. 12a that the life cycle of the $\mathrm{NAO}^{-}$event during the period from 7 to 22 February 1969 bears a striking resemblance to a blocking process driven by synoptic-scale eddies in the Atlantic sector (Butchart et al. 1989). The strongest negative NAO index values were observed on 16 February. During the life cycle of the NAO event, a meandering blocklike flow was observed in the NAO region, implicating that synoptic-scale eddies played an important role in both intensification and decay of $\mathrm{NAO}^{-}$events (blocking events) (Lupo and Bosart 1999; LLW). Figure 12b represents the life cycle of an observed $\mathrm{NAO}^{-}$event. This event was strongest on 26 December 1989. It appears that in this stage, the small-scale troughs and ridges are mostly absorbed by the zonal mean westerly flow and form an intensified westerly jet. In a word, the life processes of the two NAO events are very similar to those obtained from the weakly nonlinear NAO model in LLW.

It should be pointed out that the $\mathrm{NAO}^{-}$events meet the blocking criterion detailed in Lupo and Bosart (1999) and references therein. Thus, the $\mathrm{NAO}^{-}$events were actually identical to the blocking events in the Atlantic sector in many aspects. Notwithstanding, the time scale of $\mathrm{NAO}^{+}$events was about two weeks, and they do not meet any blocking criterion. However, this does not mean that the $\mathrm{NAO}^{+}$event is not a free mode. As we have demonstrated in the above section, an $\mathrm{NAO}^{+}$event in the planetary-scale field can be a free mode.

Figure 13 shows the scatterplots of the streamfunction against PV for two NAO cases shown in Fig. 12 in which the scatter diagrams are computed in a box region prescribed in this figure. It should be pointed out that a line is plotted in the scatter diagrams in order to reflect how the slope of the scatter diagrams changes during the NAO life cycle for its two phases. It can be shown in Fig. 13a that for the $\mathrm{NAO}^{-}$event the scatter diagrams indeed steepened during the intensification phase from 7 to 16 February, but this trend reversed during the decay phase from 16 to 22 February. The change of the observed scatter diagram was similar to the theoretical result in Fig. 9a. Nevertheless, the scat- 
(a)

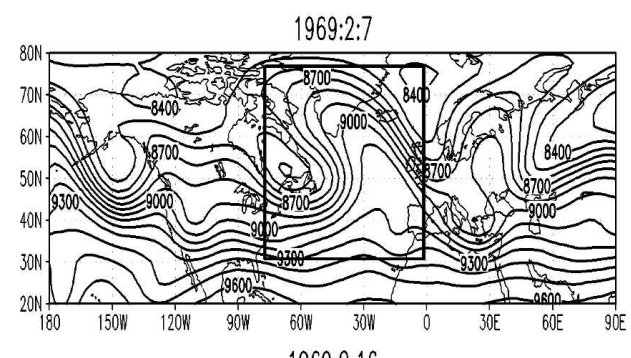

1969:2:16
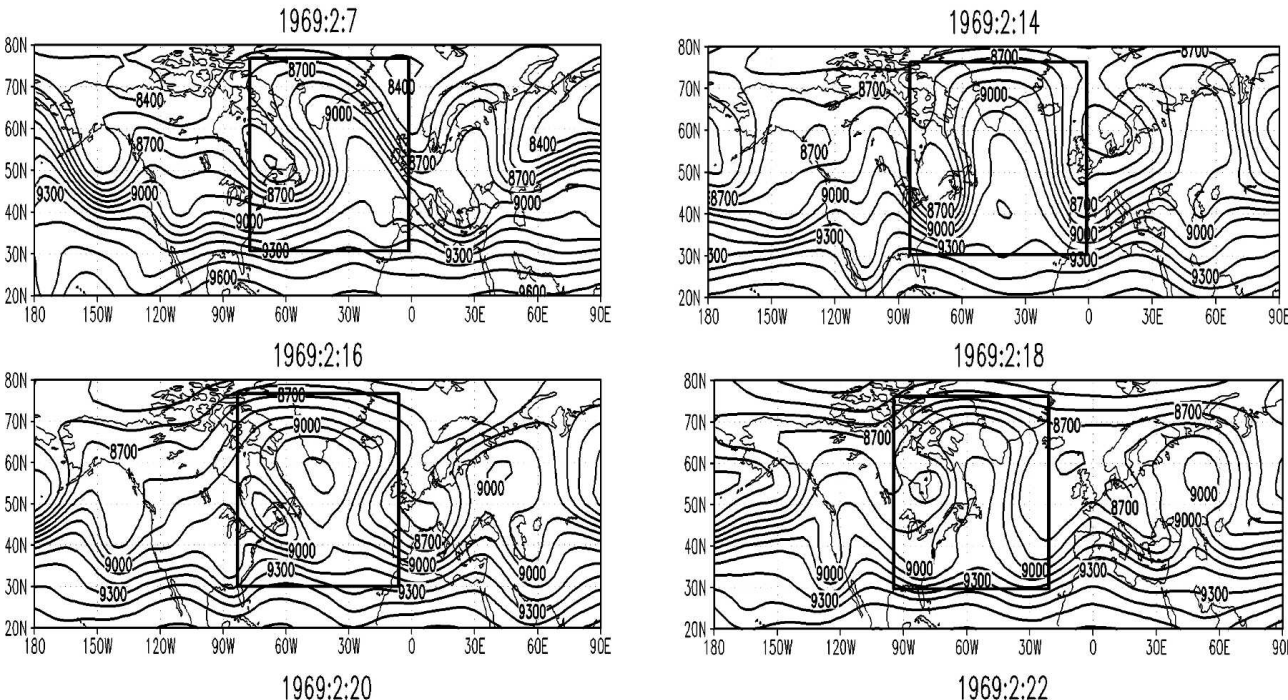

$1969: 2: 18$
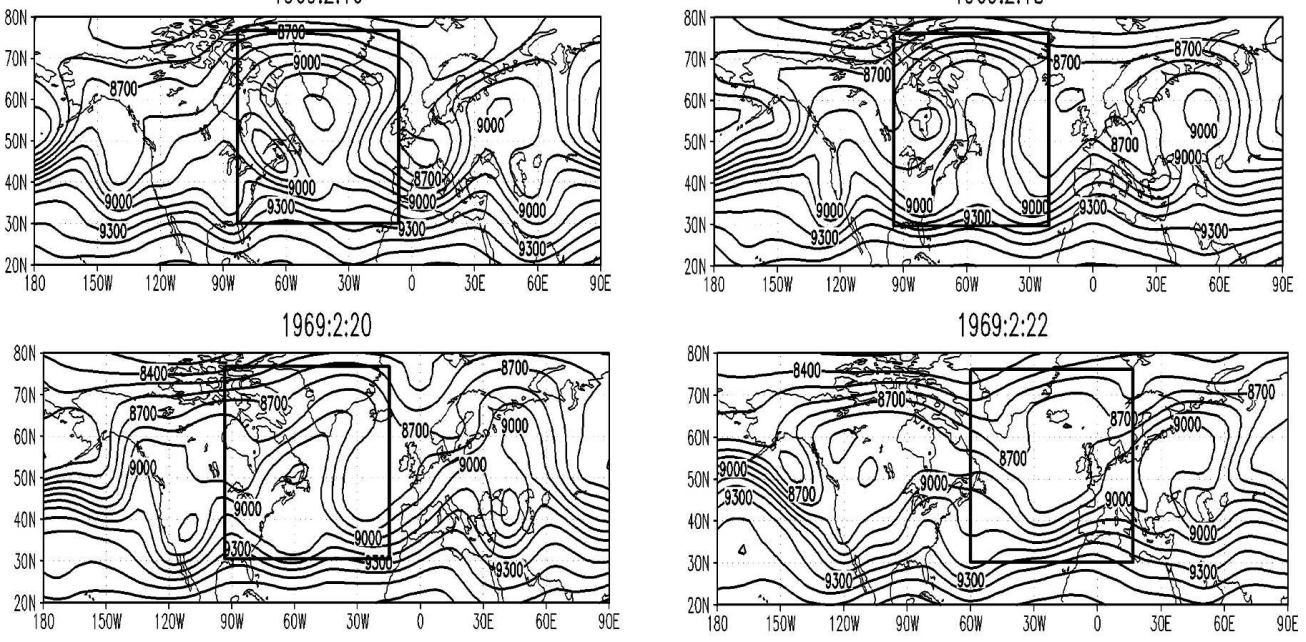

(b)
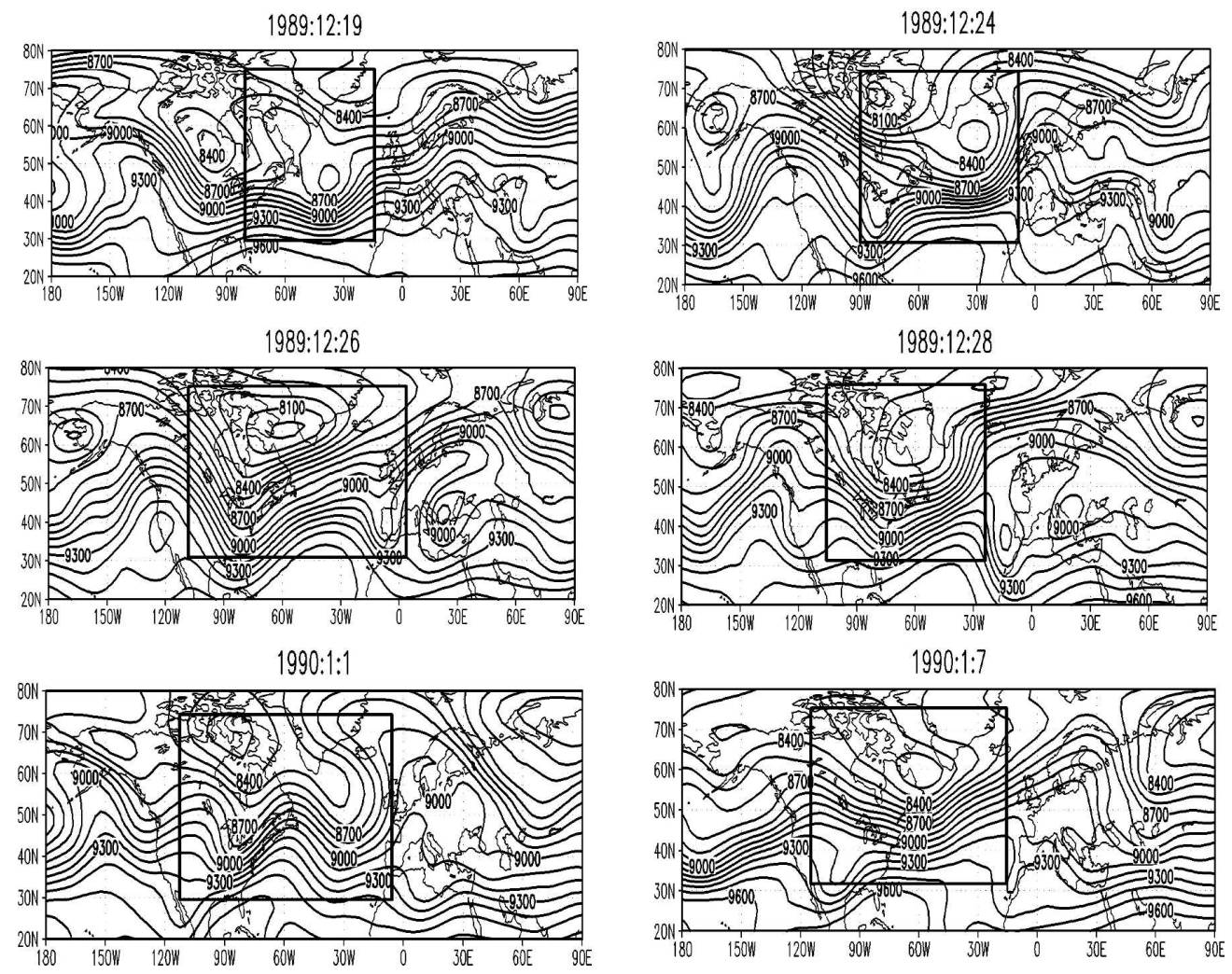

FIG. 12. Geopotential height fields at 300-mb of observed NAO events during the periods from 7 to 22 Feb 1969 and from 19 Dec 1989 to 7 Jan 1990: (a) negative phase and (b) positive phase. 
(a)
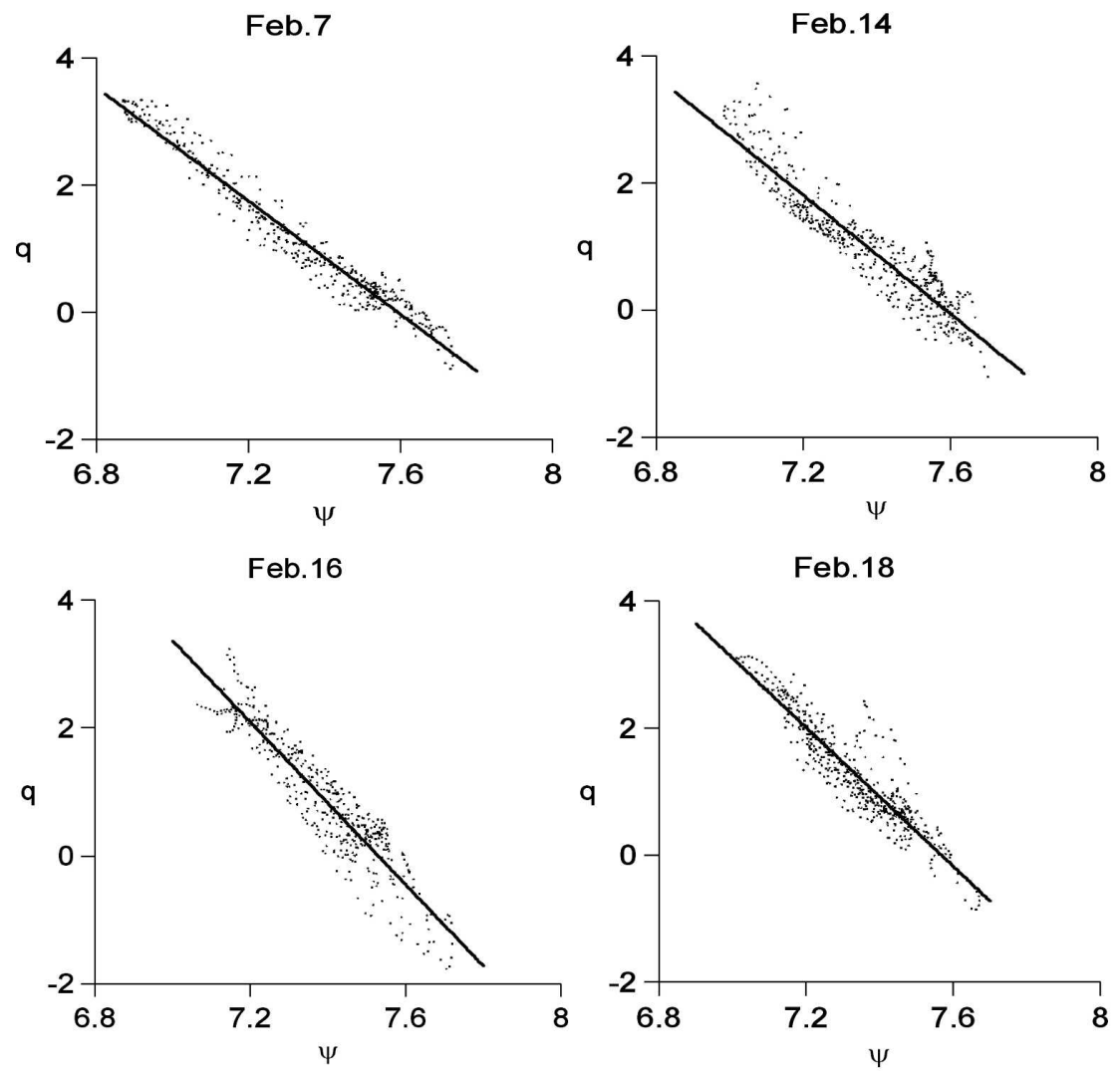

Feb.18

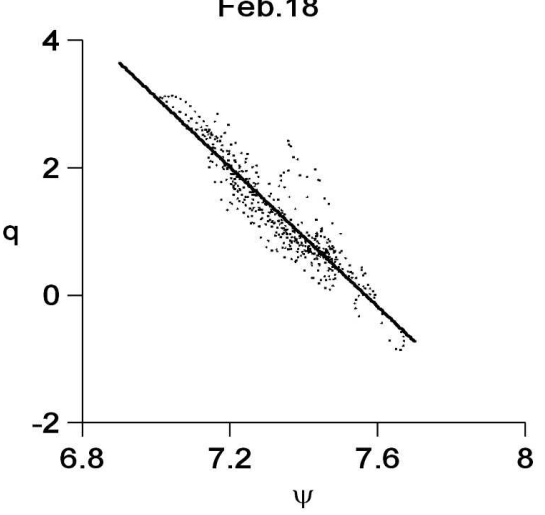

Feb.20
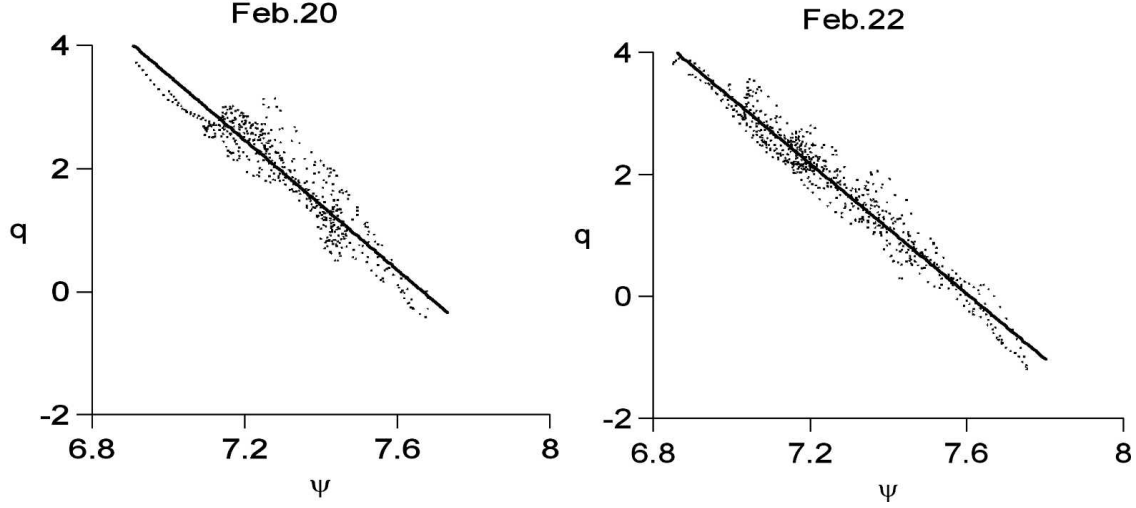

FIG. 13. Scatter diagrams of the total streamfunction $\left(10^{-4} \mathrm{~s}^{-1}\right)$ against its corresponding PV $\left(10^{8} \mathrm{~m}^{2} \mathrm{~s}^{-1}\right)$ for the observed negative- and positive-phase NAO cases in Fig. 12: (a) negative phase and (b) positive phase.

terplots in the composite field shown in Fig. 11a do not possess such a feature in that the synoptic-scale eddies almost have been filtered out. This confirms our new finding that synoptic-scale eddies can play a key role in causing the steepening and shoaling of the scatter diagrams of the streamfunction against PV associated with the life cycle of the $\mathrm{NAO}^{-}$event.

On the other hand, we see in Fig. 13b that the slope of the main straight line in the $q-\psi$ scatter diagrams for the positive-phase case shows a shoaling during the intensification stage from 19 to 26 December 1989. This tendency reversed, however, during the decay stage from 26 December 1989 to 7 January 1990. It is obvious that such a change in the scatter diagrams is very similar to theoretical behavior in Fig. 9b. Thus, it is natural to conclude from a comparison with Figs. 7, 9b, and 11b 
(b)
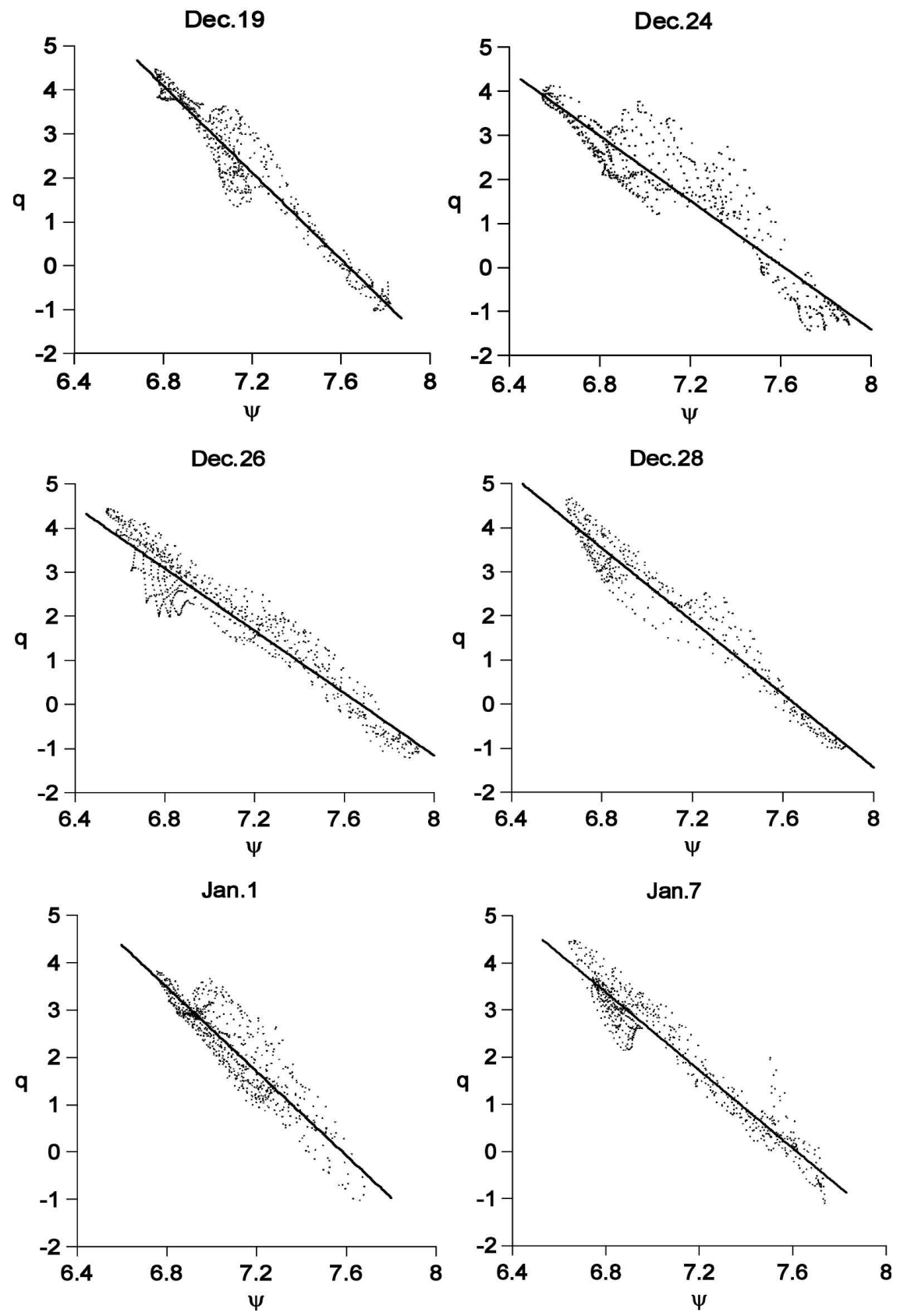

FIG. 13. (Continued)

that synoptic-scale eddies can play an important role in causing a change in the slope of the positive-phase scatter diagrams, but have a reverse role compared to the negative NAO phase.

\section{Numerical experiments and validity of the weakly nonlinear NAO model}

It can be seen using the composite of NAO events here that the $\mathrm{NAO}^{-}$and $\mathrm{NAO}^{+}$events correspond to split jet and intensified jet anomalies in the North Atlantic sector, respectively. The scatter diagrams of the streamfunction versus $\mathrm{PV}$ for $\mathrm{NAO}^{-}$and $\mathrm{NAO}^{+}$events that were obtained theoretically from LLW are consistent with the HMR numerical result although their experiment is a strongly nonlinear numerical experiment for split jet and intensified jet anomalies. The HMR numerical result, shown in Fig. 14, is cited to demonstrate the relevance of the weakly nonlinear model in 

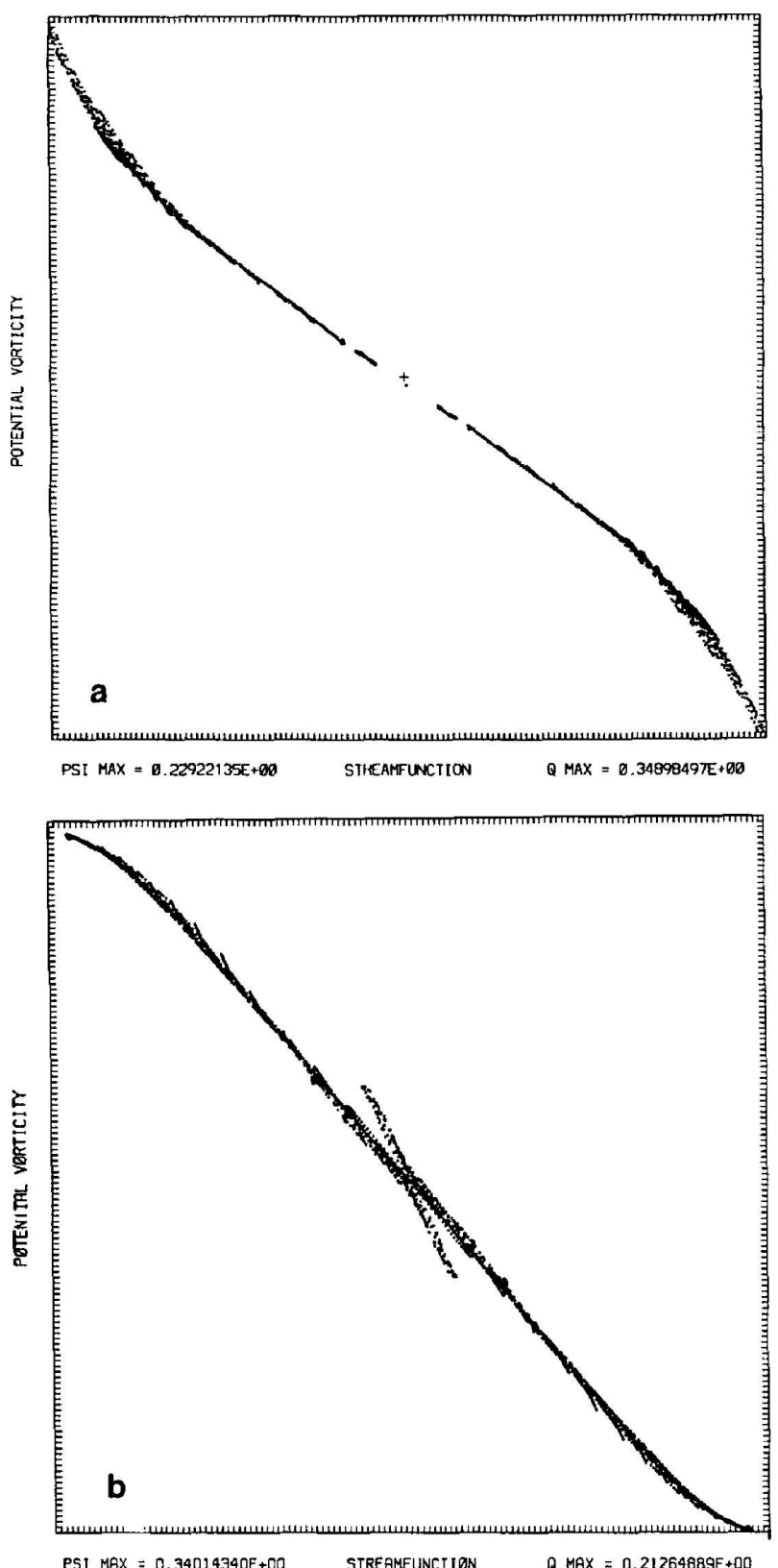

FIG. 14. Scatter diagrams of PV against streamfunction for the quasi-steady intense jet and split jet anomalies from the strongly nonlinear numerical experiment: (a) intense jet anomaly and (b) split jet anomaly (from Haines and Malanotte-Rizzoli 1991, their Fig. 14).

LLW to the free mode characteristics of $\mathrm{NAO}^{+}$and $\mathrm{NAO}^{-}$events. It is obvious that Fig. 14a looks like Fig. 8 , and that Fig. 14b is similar to Fig. 3. Figures 3 and 8 correspond to the mature $\mathrm{NAO}^{-}$and $\mathrm{NAO}^{+}$events (day 9), respectively, and bear a striking resemblance to the split jet and intense jet type anomalies. Thus, the HMR strongly nonlinear experiment sufficiently indicates that the theoretical NAO model proposed in
LLW can capture the free mode characteristics of eddydriven NAO events, even if it is a weakly nonlinear model.

\section{Conclusions and discussions}

In this paper, we have confirmed using theoretical and diagnostic evidence that the NAO events for negative and positive phases approximately correspond to free modes even though they are driven by synopticscale eddies. A time series of scatter diagrams of the streamfunction against PV are calculated for the $\mathrm{NAO}^{-}$and $\mathrm{NAO}^{+}$events obtained theoretically by LLW. It is found that the scatter diagrams of the NAO event during its life cycle display an approximately linear functional relation between the streamfunction and PV in a planetary-scale field, thus suggesting that the NAO event in its two phases is approximately a free mode.

The time series of the planetary-scale scatter diagrams for the $\mathrm{NAO}^{-}$event show a qualitative trend toward a multivalued functional relationship in a narrow region, which is particularly noticeable and more modonlike during the mature phase. Thus, this indicates that the $\mathrm{NAO}^{-}$events may correspond to isolated free modes (blocking events) in the North Atlantic sector. The slope of the main straight line in the scatter diagrams is time independent, which is also tenable for the $\mathrm{NAO}^{+}$event. But there is only a linear singlevalued functional relationship in the scatterplots of $q_{P}$ versus $\psi_{P}$ for the positive phase. A diagnostic study using the composite fields was presented to support the theoretical results.

As the synoptic-scale eddies that force NAO events to form are included in the streamfunction field, the instantaneous scatter diagrams of the total streamfunction against the corresponding PV calculated during the life cycle of the NAO event show that for the $\mathrm{NAO}^{-}$ event the scatter diagrams steepened during the intensification period. This trend reversed, however, during the decay stage. Such a characteristic is not found in previous diagnostic studies of blocking events by Butchart et al. (1989) and Ek and Swaters (1994), although they found that a steepening of the scatter diagram is in the growth stage of the blocking event. For the positive phase the slope of the main straight line in the scatter diagrams tended to shoal during the intensification phase, but this tendency reversed during the decay phase. If the synoptic-scale eddies were excluded, no steepening and shoaling are observed in the scatterplots, thus indicating that the steepening and shoaling of the scatter diagrams for two phases of the NAO event are strongly associated with synoptic-scale ed- 
dies. The diagnostic study of observed NAO cases presented here confirms this speculation.

In conclusion, although our theoretical model is weakly nonlinear, the theoretical results obtained from the weakly nonlinear model of LLW compare well with the diagnostic results using composite fields, observed NAO cases and the numerical results of HMR. In particular, the HMR strongly nonlinear numerical experiment seems to provide evidence that the free mode characteristics of the NAO events in the real atmosphere can be captured better by the weakly nonlinear model of LLW even though the real $\mathrm{NAO}^{-}$event at mature stage can correspond to a strongly nonlinear process.

However, why the slope of the main straight line in the $q_{T}\left(\psi_{T}\right)$ scatter diagrams of the $\mathrm{NAO}^{+}$event exhibited a trend opposite to that of the negative-phase event has not been explored here. In particular, why the appearance of $\mathrm{NAO}^{-}$and $\mathrm{NAO}^{+}$events sometimes alternate is also unclear (Benedict et al. 2004). Further diagnostic and theoretical studies of these events clearly are needed in the future.

Acknowledgments. The first and second authors acknowledge the support from the National Natural Science Foundation of China (4057016), National Outstanding Youth Natural Science Foundation of China (40325016), National Basic Research Program (Program 973; 2005CB422302), and FANEDD. The third author would also like to thank the support of the Missouri Research Board and U.S. Department of Energy. The authors express an appreciation to Dr. Lixin $\mathrm{Wu}$ for his comments on the original manuscript of this paper. The authors thank Dr. S. Feldstein and an anonymous reviewer for useful suggestions in improving this paper.

\section{REFERENCES}

Anderson, J. L., 1992: Barotropic stationary states and persistent anomalies in the atmospheres. J. Atmos. Sci., 49, 1709-1722.

Benedict, J. J., S. Lee, and S. B. Feldstein, 2004: Synoptic view of the North Atlantic Oscillation. J. Atmos. Sci., 61, 121-144.

Berggren, R., B. Bolin, and C. G. Rossby, 1949: An aerological study of zonal motion, its perturbations and break-down. Tellus, 1, 14-37.

Branstator, G., and J. D. Opsteegh, 1989: Free solutions of the barotropic vorticity equation. J. Atmos. Sci., 46, 1799-1814.

Butchart, N., K. Haines, and J. C. Marshall, 1989: A theoretical study of solitary waves and atmospheric blocking. J. Atmos. Sci., 46, 2063-2078.
Cash, B. A., P. J. Kushner, and G. K. Vallis, 2005: Zonal asymmetries, teleconnection, and annular patterns. J. Atmos. Sci., 62, 207-219.

Dole, R. M., 1986: Persistent anomalies of the extratropical Northern Hemisphere wintertime circulation: Structure. Mon. Wea. Rev., 114, 178-207.

— and N. D. Gordon, 1983: Persistent anomalies of the extratropical Northern Hemisphere wintertime circulation: Geophysical distribution and regional persistence characteristics. Mon. Wea. Rev., 111, 1567-1586.

Ek, N. R., and G. E. Swaters, 1994: Geostrophic scatter diagrams and the application of quasigeostrophic free-mode theory to a northeast Pacific blocking episode. J. Atmos. Sci., 51, 563581.

Feldstein, S. B., 2003: The dynamics of NAO teleconnection pattern growth and decay. Quart. J. Roy. Meteor. Soc., 129, 901924

Haines, K., and P. Malanotte-Rizzoli, 1991: Isolated anomalies in westerly jet streams: A unified approach. J. Atmos. Sci., 48, $510-526$.

,-- , and M. Morgan, 1993: Persistent jet stream intensifications: A comparison between theory and data. J. Atmos. Sci., 50, 145-154.

Häkkinen, S., 2000: Decadal air-sea interaction in the North Atlantic based on observations and modeling results. J. Climate, 13, 1195-1219.

Hurrell, J. W., 1995a: Decadal trends in the North Atlantic oscillations: Regional temperatures and precipitation. Science, 269, 676-679.

— 1995b: Transient eddy forcing of the rotational flow during northern winter. J. Atmos. Sci., 52, 2286-2301.

Luo, D., 2005: Interaction between envelope soliton vortex pair blocks and synoptic-scale eddies in an inhomogeneous baroclinicity environment. Quart. J. Roy. Meteor. Soc., 131, 125154.

— , and Z. Chen, 2006: The role of land-sea topography in blocking formation in a block-eddy interaction model. J. Atmos. Sci., 63, 3056-3065.

—, A. Lupo, and H. Wan, 2007: Dynamics of eddy-driven lowfrequency dipole modes. Part I: A simple model of North Atlantic Oscillations. J. Atmos. Sci., 64, 3-28.

Lupo, A. R., and L.F. Bosart, 1999: An analysis of a relatively rare case of continental blocking. Quart. J. Roy. Meteor. Soc., 125, 107-138.

Malguzzi, P., and P. Malanotte-Rizzoli, 1984: Nonlinear stationary Rossby waves on nonuniform zonal winds and atmospheric blocking. Part I: The analytical theory. J. Atmos. Sci., 41, 2620-2628.

McWilliams, J. C., 1980: An application of equivalent modons to atmospheric blocking. Dyn. Atmos. Oceans, 5, 43-66.

Read, P. L., P. B. Rhines, and A. A. White, 1986: Geostrophic scatter diagrams and potential vorticity dynamics. J. Atmos. Sci., 43, 3226-3240.

Vallis, G. K., E. P. Gerber, P. J. Kushner, and B. A. Cash, 2004: A mechanism and simple dynamical model of the North Atlantic Oscillation and annular modes. J. Atmos. Sci., 61, 264-280. 